\title{
A hybrid method for anisotropic elliptic problems based on the coupling of an Asymptotic-Preserving method with the Asymptotic-Limit model.
}

\author{
A. Crestetto, F. Deluzet, C. Negulescu* \\ Institut de Mathématiques de Toulouse; UMR 5219 \\ Université de Toulouse; CNRS \\ UPS IMT, 118 route de Narbonne; F-31062 Toulouse, France
}

April 27, 2022

\begin{abstract}
This paper presents a hybrid numerical method to solve efficiently a class of highly anisotropic elliptic problems. The anisotropy is aligned with one coordinateaxis and its strength is described by a parameter $\varepsilon \in(0,1]$, which can largely vary in the study domain. Our hybrid model is based on asymptotic techniques and couples (spatially) an Asymptotic-Preserving model with its asymptotic Limit model, the latter being used in regions where the anisotropy parameter $\varepsilon$ is small. Adequate coupling conditions link the two models. Aim of this hybrid procedure is to reduce the computational time for problems where the region of small $\varepsilon$ values extends over a significant part of the domain, and this due to the reduced complexity of the limit model.
\end{abstract}

Keywords : Anisotropic elliptic problem, Singular Perturbation model, Limit model, Asymptotic-Preserving scheme, Hybrid model, Dirichlet-Neumann transfer conditions.

\section{Introduction}

The present work is a contribution to the numerical resolution of highly anisotropic elliptic equations, the anisotropy being aligned with one coordinate axis and described by a perturbation parameter $\varepsilon \in(0,1]$, varying considerably in the study domain. The approach presented here is based on a coupling strategy, solving an AsymptoticPreserving reformulation of the elliptic problem where $\varepsilon$ is non-negligible and solving

\footnotetext{
*Electronic address: anais.crestetto@math.univ-toulouse.fr; fabrice.deluzet@math.univ-toulouse.fr; claudia.negulescu@math.univ-toulouse.fr
} 
the corresponding asymptotic Limit model, where $\varepsilon$ is quasi vanishing. The strategy we propose is particularly well suited for physical systems in which the anisotropy parameter $\varepsilon$ is very small in a large part of the study domain.

Such kind of directionally anisotropic diffusion systems are common in physical applications, such as plasma physics [4, 13, 14, 16, 25, 26].

The application which was at the origin of the present work comes from strongly magnetized ionospheric plasmas [1,15, 21]. The problem we shall study here is extracted from the Dynamo model and represents an elliptic equation for the computation of the electric potential in $2 \mathrm{D}$, i.e.

$$
(P) \begin{cases}-\nabla \cdot(\mathbb{A} \nabla u)=f, & \text { in } \Omega:=\Omega_{x} \times \Omega_{z}, \\ (\mathbb{A} \nabla u) \cdot n=g, & \text { on } \Omega_{x} \times \partial \Omega_{z}, \\ u=0, & \text { on } \partial \Omega_{x} \times \Omega_{z},\end{cases}
$$

where $\Omega_{x} \subset \mathbb{R}, \Omega_{z} \subset \mathbb{R}$ are intervals and $\partial \Omega$ denotes the boundary of $\Omega$, with outward normal $n$. We assume that the anisotropy direction is fixed and aligned with the $z$ coordinate, the diffusion matrix $\mathbb{A}$ being thus given by

$$
\mathbb{A}=\left(\begin{array}{cc}
A_{x} & 0 \\
0 & \frac{1}{\varepsilon} A_{z}
\end{array}\right),
$$

with $A_{x}$ and $A_{z}$ of the same order of magnitude. The high anisotropy of the problem is parametrized by $\varepsilon \in(0,1]$ that can become very small in some regions of $\Omega$. In the ionospheric plasma framework referred above, this parameter $\varepsilon$ is the ratio of the collision frequency to the cyclotron frequency. The aim of this paper is to propose a domain decomposition strategy for an efficient numerical resolution of this singularly perturbed system (1.1)-(1.2) (P-model) in situations where $\varepsilon$ undergoes large variations along the $z$ coordinate. Generalizations of this strategy to 3D problems, where $\mathbf{x}=(x, y) \in \Omega_{\mathbf{x}} \subset \mathbb{R}^{2}, A_{\mathbf{x}}(\mathbf{x}, z) \in \mathbb{R}^{2 \times 2}$, while the anisotropy is remaining aligned with the $z$-direction, are straightforward.

A naive resolution of (1.1)-(1.2) leads to an unusable scheme in the limit $\varepsilon \rightarrow 0$, because (1.1)-(1.2) degenerates into an ill-posed problem. Indeed, supposing $\varepsilon$ constant and letting it tend formally towards zero, yields the reduced model

$$
(R) \begin{cases}-\partial_{z}\left(A_{z} \partial_{z} u\right)=0, & \text { for }(x, z) \in \Omega_{x} \times \Omega_{z}, \\ \partial_{z} u=0, & \text { on } \Omega_{x} \times \partial \Omega_{z}, \\ u=0, & \text { on } \partial \Omega_{x} \times \Omega_{z} .\end{cases}
$$

All functions which are constant along the $z$-coordinate and which satisfy the boundary condition on $\partial \Omega_{x} \times \Omega_{z}$ are solutions of this ill-posed R-model. This non-uniqueness leads to an ill-conditioned linear system for the discretized P-model, when $\varepsilon \rightarrow 0$. To avoid this degeneracy, one way is to use an asymptotic-preserving AP-scheme, notion 
introduced firstly by Jin in [17]. These schemes are based on asymptotic techniques and consist in a reformulation of the singularly-perturbed problem $(P)$ into an equivalent problem $(A P)$, which in the limit $\varepsilon \rightarrow 0$ yields the "right" Limit-model, defined as the problem satisfied by the $\varepsilon \rightarrow 0$ limit of the solution-sequence $\left\{u^{\varepsilon}\right\}_{\varepsilon>0}$. In this case, the L-model is given by

$$
(L)\left\{\begin{array}{l}
-\partial_{x}\left(\overline{A_{x}} \partial_{x} u_{0}\right)=\bar{f}+\frac{g_{+}}{L_{z}}-\frac{g_{-}}{L_{z}}, \quad \text { for } x \in \Omega_{x}, \\
u_{0}\left(x_{ \pm}\right)=0
\end{array}\right.
$$

where the bars signify the average over the $z$-direction (anisotropy-direction). We refer the reader to Section 2.2 for its derivation. This AP-procedure permits to solve special types of singularly-perturbed problems, equally accurate with respect to $\varepsilon$, and in particular even its limit case $\varepsilon=0$. In recent works, such AP-schemes have been developed for highly anisotropic elliptic problems of type (1.1). In [7], Degond et al. present an AP-scheme for the $\varepsilon$-constant case, based on the decomposition of the unknown $u$ into its mean part along the $z$-direction and the fluctuation part (so-called duality-based strategy). The reformulated system is solved iteratively, starting from an approximation of the fluctuation. A direct resolution is proposed in [2,27], as well as a generalization to a variable anisotropy strength $\varepsilon(z)$, presenting steep gradients. A further extension to a variable anisotropy direction, given by a known vector field, is treated in [5], using in addition Lagrange multiplier techniques.

Aim of this paper is to increase the efficiency of the duality-based method of [2] (anisotropy aligned with one coordinate axis), in the particular case of an anisotropystrength $\varepsilon(z)$ which is very small in a large part of the domain. A full AP-scheme like in [2, 7] would be one possibility to solve such kind of problem. It appears however that in the region of small $\varepsilon$-values, solving the Limit-model is computationally more interesting in the framework of an anisotropy direction aligned with one coordinate axis. The reason for this is that the Limit-model is a lower-dimensional problem, in the present case a 1D, z-independent, elliptic problem.

These considerations lead us to the introduction of a domain decomposition strategy, where the AP-model is used where $\varepsilon(z)$ is of order one, and the L-model in the regions where $\varepsilon(z)$ is "small" enough, both models being coupled with appropriate interface conditions. Such a coupling is studied in the one-dimensional framework in [6]. We propose here to extend this coupling strategy to $2 \mathrm{D}$ problems and to provide the first analysis results.

Domain decomposition methods [23] are standard techniques to derive computationally efficient numerical schemes for problems showing different behaviors in different regions of the domain. For example in many-particle dynamics, microscopic models (Boltzmann equations, corresponding to our P-model) are coupled to macroscopic models (Euler equations, corresponding to our L-model) via appropriate interface conditions [11, 19, 20]. Usually the P-model is singularly perturbed, meaning that its asymptotic limit model is of different nature (e.g. kinetic compared to fluid models), such that the coupling of both models is very delicate. Indeed, the positioning of the interface 
requires the existence of a zone, where both models are valid and an automatic detection criteria. Moreover the design and computation of appropriate coupling conditions is generally challenging from an analytic as well as numerical point of view. In the transition from a kinetic equation to its hydrodynamic or diffusion limit, an issue has been proposed in [8,9] to avoid interface conditions. It consists in using a buffer zone, where both models have to be solved. However, it is not always possible to find such a buffer zone, where both models are accurate. Our (AP/L)-coupling strategy overcomes all these difficulties, thanks to the fact that the L-model is automatically recovered from the $\mathrm{AP}$ formulation as $\varepsilon \rightarrow 0$. In other words, the AP-model is a regular perturbation of the L-model, fact which permits their coupling via simple interface conditions. Moreover, the AP-formulation gives accurate results for all values $\varepsilon \in(0,1]$, such that the positioning of the interface is no more a problem.

The outline of this paper is the following. Section 2 presents the Singular-Perturbation P-problem, its asymptotic limit L-model and an Asymptotic-Preserving APreformulation, which was introduced in previous works (see [2,5,7]). Section 3 is devoted to the coupling of the AP-reformulation with the L-model via Dirichlet-Neumann transfer conditions. We first explain this coupling-strategy and then analyze it rigorously. We present in Section 4 the numerical discretization based on a finite element method and comment the obtained numerical results. Section 5 is devoted to some conclusions.

\section{The elliptic problem and its Asymptotic-Preser- ving reformulation}

The anisotropic, two dimensional elliptic problem we shall consider, is posed for simplicity reasons on a rectangular domain $\Omega=\Omega_{x} \times \Omega_{z}$, where $\Omega_{x}:=\left(x_{-}, x_{+}\right) \subset \mathbb{R}$ and $\Omega_{z}:=\left(z_{-}, z_{+}\right) \subset \mathbb{R}$ are two intervals of respective length $L_{x}$ and $L_{z}$.

Aim of this section is firstly to present the Singular-Perturbation problem we are interested in, then to recall the corresponding asymptotic limit model as well as an Asymptotic-Preserving reformulation, introduced in some previous works [2, 5]. For more mathematical details, we refer the reader to these works.

\subsection{The Singular-Perturbation problem: P-model}

We are interested in an efficient resolution of the following Singular-Perturbation problem

$$
(P) \begin{cases}-\partial_{x}\left(A_{x} \partial_{x} u_{\varepsilon}\right)-\partial_{z}\left(\frac{A_{z}}{\varepsilon(z)} \partial_{z} u_{\varepsilon}\right)=f, & \text { for }(x, z) \in \Omega_{x} \times \Omega_{z}, \\ \frac{A_{z}\left(x, z_{ \pm}\right)}{\varepsilon\left(z_{ \pm}\right)} \partial_{z} u_{\varepsilon}\left(x, z_{ \pm}\right)=g_{ \pm}(x), & \text { for } x \in \Omega_{x} \\ u_{\varepsilon}\left(x_{ \pm}, z\right)=0, & \text { for } z \in \Omega_{z},\end{cases}
$$

where we suppose that the coefficients and source terms satisfy the following hypotheses. 
Hypothesis A: We consider $A_{x}, A_{z} \in L^{\infty}(\Omega), f \in L^{2}(\Omega), g_{ \pm} \in L^{2}\left(\Omega_{x}\right)$ and $\varepsilon \in L^{\infty}\left(\Omega_{z}\right)$, satisfying

$0<m_{x} \leq A_{x}(x, z) \leq M_{x}, \quad 0<m_{z} \leq A_{z}(x, z) \leq M_{z}, \quad 0<\varepsilon_{\min } \leq \varepsilon(z) \leq \varepsilon_{\max } \leq 1$, with $m_{x}, m_{z}, M_{x}, M_{z}, \varepsilon_{\min }, \varepsilon_{\max }$ some given positive constants.

For the mathematical investigations of the coupling strategy presented in this paper, we shall assume more regularity on the diffusion matrices and source terms, i.e.

Hypothesis B: Additionally to Hypothesis A we shall assume that $A_{x}, A_{z} \in W^{1, \infty}(\Omega)$, $\left(f, g_{ \pm}\right) \in H^{1}(\Omega) \times H^{1}\left(\Omega_{x}\right)$ and $\varepsilon \in W^{1, \infty}\left(\Omega_{z}\right)$ is strictly increasing, with the bound $\|\varepsilon\|_{W^{1, \infty}\left(\Omega_{z}\right)} \leq \varepsilon_{M}$.

Let us now introduce the Hilbert-space

$$
\mathcal{V}:=\left\{\psi \in H^{1}(\Omega) / \psi\left(x_{ \pm}, z\right)=0 \text { for } z \in \Omega_{z}\right\},
$$

associated with the scalar product

$$
(\phi, \psi)_{\mathcal{V}}:=\left(\partial_{x} \phi, \partial_{x} \psi\right)_{L^{2}(\Omega)}+\left(\partial_{z} \phi, \partial_{z} \psi\right)_{L^{2}(\Omega)} .
$$

To simplify the notations, we shall denote in the following by $(\cdot, \cdot)_{L^{2}}$ resp. $(\cdot, \cdot)_{L_{x}^{2}}$ the corresponding scalar-products in $L^{2}(\Omega)$ resp. $L^{2}\left(\Omega_{x}\right)$. The variational formulation of problem $(\mathrm{P})$ reads then:

Find $u_{\varepsilon} \in \mathcal{V}$, such that

$$
\begin{aligned}
& \int_{\Omega} A_{x} \partial_{x} u_{\varepsilon} \partial_{x} \psi \mathrm{d} x \mathrm{~d} z+\int_{\Omega} \frac{A_{z}}{\varepsilon(z)} \partial_{z} u_{\varepsilon} \partial_{z} \psi \mathrm{d} x \mathrm{~d} z \\
& \quad=(f, \psi)_{L^{2}}+\left(g_{+}, \psi\left(\cdot, z_{+}\right)\right)_{L_{x}^{2}}-\left(g_{-}, \psi\left(\cdot, z_{-}\right)\right)_{L_{x}^{2}} \quad \forall \psi \in \mathcal{V} .
\end{aligned}
$$

Under Hypothesis A, this model admits a unique solution (Lax-Milgram), however its numerical approximation can not be computed for $\varepsilon \ll 1$, by simply discretizing (2.4). Indeed as explained in the introduction, (P) degenerates for $\varepsilon \rightarrow 0$ into an illposed problem $(\mathrm{R})$, called reduced model, leading to the inversion of an ill-conditioned linear system. This degeneracy motivates the construction of an Asymptotic-Preserving reformulation of $(\mathrm{P})$, which shall permit to get automatically the "right" asymptotic limit, when $\varepsilon$ is vanishing. To do this, we shall first identify this limit model.

\subsection{The limit model: L-model}

Let us consider in this subsection that $\varepsilon$ is constant. In the limit $\varepsilon \rightarrow 0$, the solution of the singular-perturbation model $(\mathrm{P})$ is shown to converge towards some function $u_{0}$, solution of a limit model to be identified here. This solution is a particular solution of the $\mathrm{R}$-model and it is thus independent of the $z$ variable.

To simplify the following computations, let us introduce the following notations: for a function $f$, we denote by $\bar{f}$ the average over the $z$-direction (anisotropy direction) and by $f^{\prime}$ the fluctuation part, given by

$$
\bar{f}(x):=\frac{1}{L_{z}} \int_{\Omega_{z}} f(x, z) \mathrm{d} z, \quad f^{\prime}:=f-\bar{f} .
$$


We then have the following properties:

$$
\begin{array}{lll}
\overline{f^{\prime}}=0, & \overline{\left(\frac{\partial f}{\partial x}\right)}=\frac{\partial \bar{f}}{\partial x}, & \overline{f g}=\bar{f} \bar{g}+\overline{f^{\prime} g^{\prime}} \\
\frac{\partial f}{\partial z}=\frac{\partial f^{\prime}}{\partial z}, & \left(\frac{\partial f}{\partial x}\right)^{\prime}=\partial \frac{f^{\prime}}{\partial x}, & (f g)^{\prime}=f^{\prime} g^{\prime}-\overline{f^{\prime} g^{\prime}}+\bar{f} g^{\prime}+f^{\prime} \bar{g}
\end{array}
$$

Now, integrating (2.4) along the $z$-coordinate, passing to the limit $\varepsilon \rightarrow 0$ and assuming that $u_{\varepsilon} \rightarrow_{\varepsilon \rightarrow 0} u_{0}$ in $H^{1}(\Omega)$ with $u_{0}=u_{0}(x)$, permits to obtain the system satisfied by the limit solution $u_{0}$, i.e.

$$
(L)\left\{\begin{array}{l}
-\partial_{x}\left(\overline{A_{x}} \partial_{x} u_{0}\right)=\bar{f}+\frac{g_{+}}{L_{z}}-\frac{g_{-}}{L_{z}}, \quad \text { for } x \in \Omega_{x}, \\
u_{0}\left(x_{ \pm}\right)=0 .
\end{array}\right.
$$

This so-called L-model is well-posed due to the Lax-Milgram theorem and provides an accurate solution of the P-model for very small values of $\varepsilon$.

The basic idea of Asymptotic-Preserving schemes is now to reformulate the P-model in such a manner to lead automatically towards the L-model in the limit $\varepsilon \rightarrow 0$, and not towards the ill-posed R-model (1.3). This procedure seems reasonable if one wants to treat, with no huge computational costs, problems with highly variable anisotropies $\varepsilon$ within the domain.

\subsection{The fully Asymptotic-Preserving reformulation: AP-model}

Decomposing each quantity of the P-model in its average and fluctuation part, $u_{\varepsilon}(x, z)=$ $\bar{u}_{\varepsilon}(x)+u_{\varepsilon}^{\prime}(x, z)$, permits to get an equivalent reformulation of the Singularly-Perturbed problem $(\mathrm{P})$, called Asymptotic-Preserving model (AP-model). This model consists of two sets of equations, one for each part of the solution. Its derivation is recalled in the next lines.

Taking first the mean of the P-model over $z$, we obtain the following problem, to be solved for $\bar{u}_{\varepsilon}(x)$, problem which depends on $\varepsilon$ only through the source term

$$
(\overline{A P})\left\{\begin{array}{l}
-\partial_{x}\left(\overline{A_{x}} \partial_{x} \bar{u}_{\varepsilon}\right)=\bar{f}+\frac{g_{+}}{L_{z}}-\frac{g_{-}}{L_{z}}+\partial_{x}\left(\overline{A_{x}^{\prime} \partial_{x} u_{\varepsilon}^{\prime}}\right), \quad \text { for } x \in \Omega_{x}, \\
\bar{u}_{\varepsilon}\left(x_{ \pm}\right)=0 .
\end{array}\right.
$$

Secondly, decomposing now simply $u_{\varepsilon}(x, z)=\bar{u}_{\varepsilon}(x)+u_{\varepsilon}^{\prime}(x, z)$ in $(\mathrm{P})$, yields the following equation for $u_{\varepsilon}^{\prime}(x, z)$ :

$$
\left(A P^{\prime}\right) \begin{cases}-\partial_{x}\left(A_{x} \partial_{x} u_{\varepsilon}^{\prime}\right)-\partial_{z}\left(\frac{A_{z}}{\varepsilon(z)} \partial_{z} u_{\varepsilon}^{\prime}\right)=f+\partial_{x}\left(A_{x} \partial_{x} \bar{u}_{\varepsilon}\right), & \text { for }(x, z) \in \Omega_{x} \times \Omega_{z} \\ \frac{A_{z}\left(x, z_{ \pm}\right)}{\varepsilon\left(z_{ \pm}\right)} \partial_{z} u_{\varepsilon}^{\prime}\left(x, z_{ \pm}\right)=g_{ \pm}(x), & \text { for } x \in \Omega_{x}, \\ u_{\varepsilon}^{\prime}\left(x_{ \pm}, z\right)=0, & \text { for } z \in \Omega_{z}, \\ \overline{u_{\varepsilon}^{\prime}}=0, & \text { for } x \in \Omega_{x} \text { (constraint). }\end{cases}
$$


The coupled system $(\overline{A P})-\left(A P^{\prime}\right)$ is the Duality-Based AP-reformulation introduced in [5] and shown to be completely equivalent to the P-system for fixed $\varepsilon>0$, leading however in the limit $\varepsilon \rightarrow 0$ towards the well-posed L-model. The important ingredient in this reformulation is the constraint ${\overline{u^{\prime}}}_{\varepsilon}=0$, which is automatically satisfied for $\varepsilon>0$, but helps to get in the limit $\varepsilon \rightarrow 0$ the constraint ${\overline{u^{\prime}}}_{0}=0$, which is the missing information in $(\mathrm{R})$ to get the well-posed L-model. For more details we refer the reader to [5], in particular for the rigorous existence, uniqueness and $\varepsilon \rightarrow 0$ convergence proofs.

Let us introduce the following Hilbert-space

$$
\mathcal{W}:=\left\{\psi \in H^{1}\left(\Omega_{x}\right) / \psi\left(x_{ \pm}\right)=0\right\}, \quad(\phi, \psi)_{\mathcal{W}}:=\left(\partial_{x} \phi, \partial_{x} \psi\right)_{L^{2}}
$$

Introducing a Lagrangian multiplier in order to cope with the constraint $\bar{u}_{\varepsilon}^{\prime}=0$, the variational formulation of problem $(\overline{A P})-\left(A P^{\prime}\right)$ writes:

Find $\left(\bar{u}_{\varepsilon}, u_{\varepsilon}^{\prime}, \bar{P}\right) \in \mathcal{W} \times \mathcal{V} \times L^{2}\left(\Omega_{x}\right)$ such that

$$
(A P)\left\{\begin{array}{cc}
\left(\overline{A_{x}} \partial_{x} \bar{u}_{\varepsilon}, \partial_{x} \bar{\psi}\right)_{L_{x}^{2}}=(\bar{f}, \bar{\psi})_{L_{x}^{2}}+\frac{1}{L_{z}}\left(g_{+}-g_{-}, \bar{\psi}\right)_{L_{x}^{2}}-\frac{1}{L_{z}}\left(A_{x}^{\prime} \partial_{x} u_{\varepsilon}^{\prime}, \partial_{x} \bar{\psi}\right)_{L^{2}} \\
\left(A_{x} \partial_{x} u_{\varepsilon}^{\prime}, \partial_{x} \psi^{\prime}\right)_{L^{2}}+\left(\frac{A_{z}}{\varepsilon} \partial_{z} u_{\varepsilon}^{\prime}, \partial_{z} \psi^{\prime}\right)_{L^{2}}+L_{z}\left(\bar{P}, \frac{1}{\varepsilon} \psi^{\prime}\right)_{L_{x}^{2}}=\left(f, \psi^{\prime}\right)_{L^{2}} \\
+\left(g_{+}, \psi^{\prime}\left(\cdot, z_{+}\right)\right)_{L_{x}^{2}}-\left(g_{-}, \psi^{\prime}\left(\cdot, z_{-}\right)\right)_{L_{x}^{2}}-\left(A_{x} \partial_{x} \bar{u}_{\varepsilon}, \partial_{x} \psi^{\prime}\right)_{L^{2}}, & \forall \psi^{\prime} \in \mathcal{V}, \\
\left(\bar{Q}, \overline{u_{\varepsilon}^{\prime}}\right)_{L_{x}^{2}}=0, \quad \forall \bar{Q} \in L^{2}\left(\Omega_{x}\right) . &
\end{array}\right.
$$

Solving (2.10) instead of (2.4) is numerically much more appropriate, as one gets automatically the limit problem (2.7) for vanishing $\varepsilon$, which means (2.10) is not degenerating for $\varepsilon \rightarrow 0$. However, if the computational domain contains a large region, where the value of the parameter $\varepsilon$ is very small, it can be more efficient from a computational point of view (simulation time and memory storage) to solve there directly the L-model, the latter model being of lower dimension. These considerations lead naturally to the idea of a coupling strategy between the AP-model (2.10) (in regions where $\varepsilon \sim \mathcal{O}(1)$ ) and the 1D L-model (2.7)) (where $\varepsilon \ll 1)$.

\section{The $(\mathrm{AP} / \mathrm{L})$-coupling}

This section is devoted to the coupling of the Asymptotic-Preserving reformulation (2.8)(2.9) with the asymptotic limit model (2.7). Firstly we shall explain the strategy and then analyze it rigorously. For simplicity reasons, we shall omit in the following the index $\varepsilon$ of the unknown $u_{\varepsilon}$.

Let us remark here that a coupling of the P-model with the L-model, which is usually done in literature [11, 19, 20], is not always possible. Indeed, one important issue in developing a method implementing a standard discretization of the $(\mathrm{P})$-model in a subdomain and the $(\mathrm{L})$-model elsewhere requires that both models are solved in a common 
region containing the coupling interface. However, the limit model defines an accurate approximation for small values of the asymptotic parameter while the $(\mathrm{P})$-model suffers from significant precision discrepancies for these same parameter values. Defining a region where both models can produce an accurate approximation of the solution at the same time may thus be impossible (see [5, Figure 3.3] for an illustration). In contrast to this, the AP-formulation gives accurate results for all values of $\varepsilon \in[0,1]$, such that a coupling is always possible. Furthermore, due to the fact that the AP-formulation is a regular perturbation of the L-model, coupling interface conditions are easy to design. All these arguments underline the importance of the here presented strategy.

\subsection{Presentation of the coupling strategy}

Let us assume that in a large region of the computational domain the anisotropy parameter $\varepsilon$ is very small. For simplicity reasons, we assume that $\varepsilon$ is a strictly increasing function and that the domain is decomposed in the $z$-direction into two sub-domains, delimited by an interface $z_{\iota} \in\left(z_{-}, z_{+}\right)$. Let us introduce the following notation $\Omega_{z}^{1}:=\left(z_{\iota}, z_{+}\right)$, $\Omega_{z}^{2}:=\left(z_{-}, z_{\iota}\right), \Omega_{1}:=\Omega_{x} \times \Omega_{z}^{1}$ and $\Omega_{2}:=\Omega_{x} \times \Omega_{z}^{2}$, and assume $\varepsilon$ to be small in $\Omega_{2}$, as illustrated in Figure 1. Recall however that $\varepsilon(\cdot) \in W^{1, \infty}\left(\Omega_{z}\right)$, such that the derivatives of $\varepsilon(\cdot)$ have to be bounded, even when $\varepsilon(\cdot)$ goes to zero in some parts of the domain. Our approach is now to use in $\Omega_{2}$ the Limit-model and elsewhere the AP-model, with the aim to reduce the overall computational costs for the resolution of (1.1). Let us detail here the coupling strategy and its variational formulation.

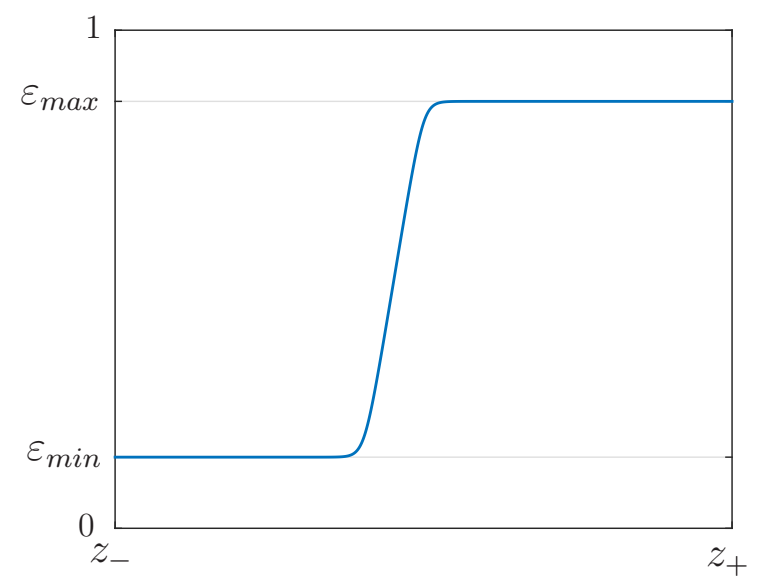

Figure 1: Illustration of the anisotropy ratio $\varepsilon$ as a function of $z$.

\subsubsection{The coupling strategy}

Let us decompose the unknown $u$ as follows $u=\bar{u}+u_{1}^{\prime}+u_{2}^{\prime}$, where

$$
u_{1}^{\prime}:=u^{\prime} \mathbb{1}_{\Omega_{x} \times \Omega_{z}^{1}} \quad \text { and } \quad u_{2}^{\prime}:=u^{\prime} \mathbb{1}_{\Omega_{x} \times \Omega_{z}^{2}}
$$

are the restrictions of the fluctuation on the two sub-domains. As mentioned above, we shall suppose that in $\Omega_{2}$ the parameter $\varepsilon$ is very small, meaning that $\varepsilon\left(z_{\iota}\right) \ll 1$. Let us now rewrite the AP-model for this new decomposition $\left(\bar{u}, u_{1}^{\prime}, u_{2}^{\prime}\right)$ coupling the two 
sub-domains via Dirichlet-Neumann transfer conditions, which translate the fact that the solution as well as its normal derivative $\partial_{z} u$ are continuous at the interface $z_{\iota}$. This new system, called in the following (AP/AP)-reformulation is completely equivalent to the AP-model (2.8)-(2.9) and is given by

$$
\begin{aligned}
& (\overline{A P})\left\{\begin{array}{l}
-\partial_{x}\left(\overline{A_{x}} \partial_{x} \bar{u}\right)=\bar{f}+\frac{g_{+}}{L_{z}}-\frac{g_{-}}{L_{z}} \\
\quad+\frac{1}{L_{z}} \partial_{x}\left(\int_{\Omega_{z}^{1}} A_{x}^{\prime} \partial_{x} u_{1}^{\prime} \mathrm{d} z+\int_{\Omega_{z}^{2}} A_{x}^{\prime} \partial_{x} u_{2}^{\prime} \mathrm{d} z\right), \quad \text { for } x \in \Omega_{x}, \\
\bar{u}\left(x_{ \pm}\right)=0,
\end{array}\right. \\
& \left(A P_{1}^{\prime}\right) \begin{cases}-\partial_{x}\left(A_{x} \partial_{x} u_{1}^{\prime}\right)-\partial_{z}\left(\frac{A_{z}}{\varepsilon(z)} \partial_{z} u_{1}^{\prime}\right)=f+\partial_{x}\left(A_{x} \partial_{x} \bar{u}\right), & \text { for }(x, z) \in \Omega_{1}, \\
\frac{A_{z}\left(x, z_{+}\right)}{\varepsilon\left(z_{+}\right)} \partial_{z} u_{1}^{\prime}\left(x, z_{+}\right)=g_{+}(x), & \text { for } x \in \Omega_{x}, \\
u_{1}^{\prime}\left(x_{ \pm}, z\right)=0, & \text { for } z \in \Omega_{z}^{1}, \\
\frac{A_{z}\left(x, z_{\iota}\right)}{\varepsilon\left(z_{\iota}\right)} \partial_{z} u_{1}^{\prime}\left(x, z_{\iota}\right)=\frac{A_{z}\left(x, z_{\iota}\right)}{\varepsilon\left(z_{\iota}\right)} \partial_{z} u_{2}^{\prime}\left(x, z_{\iota}\right), & \text { for } x \in \Omega_{x} \text { (DN-cond) } \\
\int_{\Omega_{z}^{1}} u_{1}^{\prime}(x, z) \mathrm{d} z+\int_{\Omega_{z}^{2}} u_{2}^{\prime}(x, z) \mathrm{d} z=0, & \text { for } x \in \Omega_{x} \text { (constraint), }\end{cases} \\
& \left(A P_{2}^{\prime}\right) \begin{cases}-\partial_{x}\left(A_{x} \partial_{x} u_{2}^{\prime}\right)-\partial_{z}\left(\frac{A_{z}}{\varepsilon(z)} \partial_{z} u_{2}^{\prime}\right)=f+\partial_{x}\left(A_{x} \partial_{x} \bar{u}\right), & \text { for }(x, z) \in \Omega_{2}, \\
\frac{A_{z}\left(x, z_{-}\right)}{\varepsilon\left(z_{-}\right)} \partial_{z} u_{2}^{\prime}\left(x, z_{-}\right)=g_{-}(x), & \text { for } x \in \Omega_{x} \\
u_{2}^{\prime}\left(x_{ \pm}, z\right)=0, & \text { for } z \in \Omega_{z}^{2}, \\
u_{2}^{\prime}\left(x, z_{\iota}\right)=u_{1}^{\prime}\left(x, z_{\iota}\right), & \text { for } x \in \Omega_{x} \text { (DN-cond). }\end{cases}
\end{aligned}
$$

For the mathematical and numerical study, we shall need the weak form of this system. In this aim, and in order to get rid of the non-homogeneous Dirichlet interface condition, it will be more convenient to introduce the new variable (lifting procedure)

$$
\xi_{2}^{\prime}(x, z):=u_{2}^{\prime}(x, z)-u_{1}^{\prime}\left(x, z_{\iota}\right) \quad \text { for }(x, z) \in \Omega_{x} \times \Omega_{z}^{2}
$$

where we remark that $u_{1}^{\prime}\left(x, z_{\iota}\right) \in H^{1}\left(\Omega_{x}\right)$, as by Hyp. B, the solution $u$ of the P-model belongs to $H^{2}(\Omega)$. This leads to the completely equivalent formulation, called in the 
following also (AP/AP)-reformulation, for the unknowns $\left(\bar{u}, u_{1}^{\prime}, \xi_{2}^{\prime}\right)$ :

$$
\begin{aligned}
& (\overline{A P})\left\{\begin{array}{c}
-\partial_{x}\left(\overline{A_{x}} \partial_{x} \bar{u}\right)=\bar{f}+\frac{g_{+}}{L_{z}}-\frac{g_{-}}{L_{z}}+\frac{1}{L_{z}} \partial_{x}\left(\int_{\Omega_{z}^{1}} A_{x}^{\prime} \partial_{x} u_{1}^{\prime} \mathrm{d} z\right. \\
\left.\quad+\int_{\Omega_{z}^{2}} A_{x}^{\prime} \partial_{x} u_{1}^{\prime}\left(x, z_{\iota}\right) \mathrm{d} z+\int_{\Omega_{z}^{2}} A_{x}^{\prime} \partial_{x} \xi_{2}^{\prime} \mathrm{d} z\right), \quad \text { for } x \in \Omega_{x}, \\
\bar{u}\left(x_{ \pm}\right)=0,
\end{array}\right. \\
& \left(A P_{1}^{\prime}\right) \begin{cases}-\partial_{x}\left(A_{x} \partial_{x} u_{1}^{\prime}\right)-\partial_{z}\left(\frac{A_{z}}{\varepsilon(z)} \partial_{z} u_{1}^{\prime}\right)=f+\partial_{x}\left(A_{x} \partial_{x} \bar{u}\right), & \text { for }(x, z) \in \Omega_{x} \times \Omega_{z}^{1}, \\
\frac{A_{z}\left(x, z_{+}\right)}{\varepsilon\left(z_{+}\right)} \partial_{z} u_{1}^{\prime}\left(x, z_{+}\right)=g_{+}(x), & \text { for } x \in \Omega_{x}, \\
u_{1}^{\prime}\left(x_{ \pm}, z\right)=0, & \text { for } z \in \Omega_{z}^{1}, \\
\frac{A_{z}\left(x, z_{\iota}\right)}{\varepsilon\left(z_{\iota}\right)} \partial_{z} u_{1}^{\prime}\left(x, z_{\iota}\right)=\frac{A_{z}\left(x, z_{\iota}\right)}{\varepsilon\left(z_{\iota}\right)} \partial_{z} \xi_{2}^{\prime}\left(x, z_{\iota}\right), & \text { for } x \in \Omega_{x} \\
\int_{\Omega_{z}^{1}} u_{1}^{\prime}(x, z) \mathrm{d} z+L_{z}^{2} u_{1}^{\prime}\left(x, z_{\iota}\right)+\int_{\Omega_{z}^{2}} \xi_{2}^{\prime}(x, z) \mathrm{d} z=0, & \text { for } x \in \Omega_{x} \text { (constraint), }\end{cases} \\
& \left(A P_{2}^{\prime}\right) \begin{cases}-\partial_{x}\left(A_{x} \partial_{x} \xi_{2}^{\prime}\right)-\partial_{z}\left(\frac{A_{z}}{\varepsilon(z)} \partial_{z} \xi_{2}^{\prime}\right)=f & \\
\quad+\partial_{x}\left(A_{x} \partial_{x} u_{1}^{\prime}\left(x, z_{\iota}\right)\right)+\partial_{x}\left(A_{x} \partial_{x} \bar{u}\right), & \text { for }(x, z) \in \Omega_{x} \times \Omega_{z}^{2}, \\
\frac{A_{z}\left(x, z_{-}\right)}{\varepsilon\left(z_{-}\right)} \partial_{z} \xi_{2}^{\prime}\left(x, z_{-}\right)=g_{-}(x), & \text { for } x \in \Omega_{x}, \\
\xi_{2}^{\prime}\left(x_{ \pm}, z\right)=0, & \text { for } z \in \Omega_{z}^{2}, \\
\xi_{2}^{\prime}\left(x, z_{\iota}\right)=0, & \text { for } x \in \Omega_{x} .\end{cases}
\end{aligned}
$$

We shall prove in Section 3.2 that in the limit of vanishing anisotropy strength in $\Omega_{2}$, 
i.e. $\delta=\varepsilon\left(z_{\iota}\right) \rightarrow 0$, this system yields the following hybrid-system, called (AP/L)-model:

$$
\begin{aligned}
& (\overline{A P}) \begin{cases}-\partial_{x}\left(\overline{A_{x}} \partial_{x} \bar{u}\right)=\bar{f}+\frac{g_{+}}{L_{z}}-\frac{g_{-}}{L_{z}}+\frac{1}{L_{z}} \partial_{x}\left(\int_{\Omega_{z}^{1}} A_{x}^{\prime} \partial_{x} u_{1}^{\prime} \mathrm{d} z\right. & \\
\left.\quad+\int_{\Omega_{z}^{2}} A_{x}^{\prime} \partial_{x} u_{1}^{\prime}\left(x, z_{\iota}\right) \mathrm{d} z\right), & \text { for } x \in \Omega_{x} \\
\bar{u}\left(x_{ \pm}\right)=0 & \end{cases} \\
& \left(A P_{1}^{\prime}\right) \begin{cases}-\partial_{x}\left(A_{x} \partial_{x} u_{1}^{\prime}\right)-\partial_{z}\left(\frac{A_{z}}{\varepsilon(z)} \partial_{z} u_{1}^{\prime}\right)=f+\partial_{x}\left(A_{x} \partial_{x} \bar{u}\right), & \text { for }(x, z) \in \Omega_{x} \times \Omega_{z}^{1}, \\
\frac{A_{z}\left(x, z_{+}\right)}{\varepsilon\left(z_{+}\right)} \partial_{z} u_{1}^{\prime}\left(x, z_{+}\right)=g_{+}(x), & \text { for } x \in \Omega_{x}, \\
u_{1}^{\prime}\left(x_{ \pm}, z\right)=0, & \text { for } z \in \Omega_{z}^{1}, \\
\frac{A_{z}\left(x, z_{\iota}\right)}{\varepsilon\left(z_{\iota}\right)} \partial_{z} u_{1}^{\prime}\left(x, z_{\iota}\right)=0, & \text { for } x \in \Omega_{x} \\
\int_{\Omega_{z}^{1}} u_{1}^{\prime}(x, z) \mathrm{d} z+L_{z}^{2} u_{1}^{\prime}\left(x, z_{\iota}\right)=0, & \text { for } x \in \Omega_{x} \text { (constraint), }\end{cases} \\
& \text { (L) }\left\{\xi_{2}^{\prime}(x)=0, \quad \text { for } x \in \Omega_{x} .\right.
\end{aligned}
$$

Formally, one can immediately observe that the solution $\xi_{2}^{\prime}$ of $\left(A P_{2}^{\prime}\right)$ converges (in some sense to be defined later on) in the limit $\varepsilon\left(z_{\iota}\right) \rightarrow 0$ towards zero. Indeed, letting formally $\delta=\varepsilon\left(z_{\iota}\right) \rightarrow 0$ implies that the limit function $\xi_{2}^{\prime}$ is independent of $z$. The $x$-dependence is then given by the Dirichlet interface condition in $z=z_{\iota}$, hence $\xi_{2}^{\prime} \equiv 0$ in the limit $\varepsilon\left(z_{\iota}\right) \rightarrow 0$.

The rigorous mathematical study of the approximation error introduced by using (3.14) instead of (3.13) if $\varepsilon\left(z_{\iota}\right) \ll 1$, will be the subject of Section 3.2, and shall permit to find a criterion determining where to put the interface. Aim of this paper is to show that using the hybrid AP/L-model instead of the fully AP-model is computationally more efficient when the parameter $\varepsilon(z)$ is small in a large part of the domain.

\subsubsection{Variational formulations}

Our numerical simulations will be based on finite element discretizations of the former systems. For this, we shall introduce now the variational formulations of the (AP/AP)model (3.13) as well as of the (AP/L)-model (3.14), and define the Hilbert-spaces

$$
\begin{aligned}
& \mathcal{V}_{1}:=\left\{\psi(\cdot, \cdot) \in H^{1}\left(\Omega_{1}\right) / \psi\left(x_{ \pm}, \cdot\right)=0\right\} \\
& \mathcal{V}_{2}:=\left\{\psi(\cdot, \cdot) \in H^{1}\left(\Omega_{2}\right) / \psi\left(x_{ \pm}, \cdot\right)=0, \psi\left(\cdot, z_{\iota}\right)=0\right\} \\
& \mathcal{W}:=\left\{\psi(\cdot) \in H^{1}\left(\Omega_{x}\right) / \psi\left(x_{ \pm}\right)=0\right\}
\end{aligned}
$$

associated to the scalar products

$$
\begin{aligned}
(\phi, \psi)_{\mathcal{V}_{1}} & :=\left(\partial_{x} \phi, \partial_{x} \psi\right)_{L_{1}^{2}}+\left(\partial_{z} \phi, \partial_{z} \psi\right)_{L_{1}^{2}} \\
(\phi, \psi)_{\mathcal{V}_{2}} & :=\left(\partial_{x} \phi, \partial_{x} \psi\right)_{L_{2}^{2}}+\left(\partial_{z} \phi, \partial_{z} \psi\right)_{L_{2}^{2}} \\
(\phi, \psi)_{\mathcal{W}} & :=\left(\partial_{x} \phi, \partial_{x} \psi\right)_{L_{x}^{2}}
\end{aligned}
$$


where we denoted by $(\cdot, \cdot)_{L_{1}^{2}}$ resp. $(\cdot, \cdot)_{L_{2}^{2}}$ the $L^{2}$ scalar-products in $\Omega_{1}$ resp. $\Omega_{2}$. To simplify the writing, we define further for $v^{\prime} \in \mathcal{V}_{\star}, \psi^{\prime} \in \mathcal{V}_{\star}, \bar{v} \in \mathcal{W}, \bar{\psi} \in \mathcal{W}, \bar{P} \in L^{2}\left(\Omega_{x}\right)$ and $\bar{Q} \in L^{2}\left(\Omega_{x}\right)$, the following bilinear forms

$$
\begin{aligned}
& a_{z \star}\left(v^{\prime}, \psi^{\prime}\right) \quad:=\int_{\Omega_{z}^{\star}} \int_{\Omega_{x}} \frac{A_{z}}{\varepsilon(z)} \partial_{z} v^{\prime} \partial_{z} \psi^{\prime} \mathrm{d} x \mathrm{~d} z, \\
& a_{x f_{\star}}\left(v^{\prime}, \psi^{\prime}\right):=\int_{\Omega_{z}^{\star}} \int_{\Omega_{x}} A_{x} \partial_{x} v^{\prime} \partial_{x} \psi^{\prime} \mathrm{d} x \mathrm{~d} z, \quad \quad a_{x a}(\bar{v}, \bar{\psi}):=\int_{\Omega_{x}} \overline{A_{x}} \partial_{x} \bar{v} \partial_{x} \bar{\psi} \mathrm{d} x, \\
& b_{l 1}\left(\bar{P}, \psi^{\prime}\right) \quad:=\int_{\Omega_{x}} \bar{P} \int_{\Omega_{z}^{1}} \frac{1}{\varepsilon(z)} \psi^{\prime} \mathrm{d} z \mathrm{~d} x, \quad \quad b_{c \star}\left(v^{\prime}, \bar{Q}\right):=\frac{1}{L_{z}} \int_{\Omega_{x}} \bar{Q} \int_{\Omega_{z}^{\star}} v^{\prime} \mathrm{d} z \mathrm{~d} x, \\
& c_{f \star}\left(\bar{v}, \psi^{\prime}\right) \quad:=\int_{\Omega_{z}^{\star}} \int_{\Omega_{x}} A_{x} \partial_{x} \bar{v} \partial_{x} \psi^{\prime} \mathrm{d} x \mathrm{~d} z, \quad \quad c_{a \star}\left(v^{\prime}, \bar{\psi}\right):=\int_{\Omega_{z}^{\star}} \int_{\Omega_{x}} A_{x}^{\prime} \partial_{x} v^{\prime} \partial_{x} \bar{\psi} \mathrm{d} x \mathrm{~d} z, \\
& d_{\iota}\left(v^{\prime}, \psi_{1}^{\prime}\right) \quad:=\int_{\Omega_{x}} \frac{A_{z}\left(x, z_{\iota}\right)}{\varepsilon\left(z_{\iota}\right)} \partial_{z} v^{\prime}\left(x, z_{\iota}\right) \psi_{1}^{\prime}\left(x, z_{\iota}\right) \mathrm{d} x, \quad v^{\prime} \text { s.t. } \quad \frac{A_{z}\left(\cdot, z_{\iota}\right)}{\varepsilon\left(z_{\iota}\right)} \partial_{z} v^{\prime}\left(\cdot, z_{\iota}\right) \in L^{2}\left(\Omega_{x}\right) \text {, }
\end{aligned}
$$

where $\star$ takes the value 1 or 2 . The variational formulation of the (AP/AP)-model (3.13) writes: Find $\left(\bar{u}, u_{1}^{\prime}, \xi_{2}^{\prime}, \bar{P}\right) \in \mathcal{W} \times \mathcal{V}_{1} \times \mathcal{V}_{2} \times L^{2}\left(\Omega_{x}\right)$ such that

$$
(A P / A P)\left\{\begin{array}{c}
a_{x a}(\bar{u}, \bar{\psi})=(\bar{f}, \bar{\psi})_{L_{x}^{2}}+\frac{1}{L_{z}}\left(g_{+}-g_{-}, \bar{\psi}\right)_{L_{x}^{2}} \\
-\frac{1}{L_{z}}\left(c_{a 1}\left(u_{1}^{\prime}, \bar{\psi}\right)+c_{a 2}\left(u_{1}^{\prime}\left(\cdot, z_{\iota}\right), \bar{\psi}\right)+c_{a 2}\left(\xi_{2}^{\prime}, \bar{\psi}\right)\right), \quad \forall \bar{\psi} \in \mathcal{W}, \\
a_{x f 1}\left(u_{1}^{\prime}, \psi_{1}^{\prime}\right)+a_{z 1}\left(u_{1}^{\prime}, \psi_{1}^{\prime}\right)+b_{l 1}\left(\bar{P}, \psi_{1}^{\prime}\right)=\left(f, \psi_{1}^{\prime}\right)_{L_{1}^{2}} \\
+\left(g_{+}, \psi_{1}^{\prime}\left(\cdot, z_{+}\right)\right)_{L_{x}^{2}}-d_{\iota}\left(\xi_{2}^{\prime}, \psi_{1}^{\prime}\right)-c_{f 1}\left(\bar{u}, \psi_{1}^{\prime}\right), \quad \forall \psi_{1}^{\prime} \in \mathcal{V}_{1}, \\
a_{x f 2}\left(\xi_{2}^{\prime}, \psi_{2}^{\prime}\right)+a_{z 2}\left(\xi_{2}^{\prime}, \psi_{2}^{\prime}\right)=\left(f, \psi_{2}^{\prime}\right)_{L_{2}^{2}}-\left(g_{-}, \psi_{2}^{\prime}\left(\cdot, z_{-}\right)\right)_{L_{x}^{2}}-c_{f 2}\left(\bar{u}, \psi_{2}^{\prime}\right) \\
-a_{x f 2}\left(u_{1}^{\prime}\left(\cdot, z_{\iota}\right), \psi_{2}^{\prime}\right), \quad \forall \psi_{2}^{\prime} \in \mathcal{V}_{2}, \quad \\
b_{c 1}\left(u_{1}^{\prime}, \bar{Q}\right)=-b_{c 2}\left(u_{1}^{\prime}\left(\cdot, z_{\iota}\right), \bar{Q}\right)-b_{c 2}\left(\xi_{2}^{\prime}, \bar{Q}\right), \quad \forall \bar{Q} \in L^{2} .
\end{array}\right.
$$

Equally, we obtain the following variational formulation for the (AP/L)-model (3.14): Find $\left(\bar{u}, u_{1}^{\prime}, \bar{P}\right) \in \mathcal{W} \times \mathcal{V}_{1} \times L^{2}\left(\Omega_{x}\right)$ such that

$$
(A P / L)\left\{\begin{array}{cl}
a_{x a}(\bar{u}, \bar{\psi})=(\bar{f}, \bar{\psi})_{L_{x}^{2}}+\frac{1}{L_{z}}\left(g_{+}-g_{-}, \bar{\psi}\right)_{L_{x}^{2}} & \\
-\frac{1}{L_{z}}\left(c_{a 1}\left(u_{1}^{\prime}, \bar{\psi}\right)+c_{a 2}\left(u_{1}^{\prime}\left(\cdot, z_{\iota}\right), \bar{\psi}\right)\right), & \forall \bar{\psi} \in \mathcal{W}, \\
a_{x f 1}\left(u_{1}^{\prime}, \psi_{1}^{\prime}\right)+a_{z 1}\left(u_{1}^{\prime}, \psi_{1}^{\prime}\right)+b_{l 1}\left(\bar{P}, \psi_{1}^{\prime}\right) & \\
=\left(f, \psi_{1}^{\prime}\right)_{L_{1}^{2}}+\left(g_{+}, \psi_{1}^{\prime}\left(\cdot, z_{+}\right)\right)_{L_{x}^{2}}-c_{f 1}\left(\bar{u}, \psi_{1}^{\prime}\right), & \forall \psi_{1}^{\prime} \in \mathcal{V}_{1}, \\
b_{c 1}\left(u_{1}^{\prime}, \bar{Q}\right)=-b_{c 2}\left(u_{1}^{\prime}\left(\cdot, z_{\iota}\right), \bar{Q}\right), & \forall \bar{Q} \in L^{2}\left(\Omega_{x}\right) .
\end{array}\right.
$$


It will be this (AP/L)-model which will be used in our numerical simulations (Section 4) in order to compute the solution of the highly anisotropic elliptic equation (1.1).

\subsection{Mathematical study}

Aim of this section is to study the approximation error introduced when using the Limitmodel in the sub-domain $\Omega_{x} \times \Omega_{z}^{2}$ (where the anisotropy strength $\varepsilon$ is small) instead of the original problem. In other words we are interested in the error between the solution of the (AP/AP)-model and the (AP/L)-model. This error will depend on the position of the coupling interface, more precisely on $\varepsilon\left(z_{\iota}\right)$.

Let us denote in the following by $\left(\bar{u}, u_{1}^{\prime}, \xi_{2}^{\prime}\right) \in \mathcal{W} \times \mathcal{V}_{1} \times \mathcal{V}_{2}$ the solution of (3.13) or (3.16) and by $\left(\bar{v}, v_{1}^{\prime}, 0\right) \in \mathcal{W} \times \mathcal{V}_{1} \times \mathcal{V}_{2}$ the solution of (3.14) or (3.17). Remark that the existence and uniqueness of a solution of (3.13) can be straightforwardly shown by equivalence with the AP-model (2.8)-(2.9) . Moreover the existence and uniqueness of a solution of (3.14) is shown by standard arguments, proving the boundedness of $\left(\bar{u}, u_{1}^{\prime}, \xi_{2}^{\prime}\right)$ in $\mathcal{W} \times \mathcal{V}_{1} \times \mathcal{V}_{2}$, uniformly in $\varepsilon\left(z_{\iota}\right)$, inducing thus weak convergences and passing finally to the limit in the variational formulation. We leave the details to the reader and prefer

to concentrate on the error estimate. We will begin with estimating firstly $\xi_{2}^{\prime}$, showing its convergence towards zero for $\varepsilon\left(z_{\iota}\right) \rightarrow 0$ (Theorem 3.2). Using this convergence result, we shall estimate in a second step the errors $\|\bar{u}-\bar{v}\|_{\mathcal{W}}$ as well as $\left\|u_{1}^{\prime}-v_{1}^{\prime}\right\|_{\mathcal{V}_{1}}$ (Theorem 3.3). For the convergence of $\xi_{2}^{\prime}$ we will need the following regularity result.

Lemma 3.1 Under Hypothesis B, the solution $u$ of the P-model (2.4) belongs to $H^{2}(\Omega)$ and one has the following estimates

$$
\|u\|_{H^{2}(\Omega)}+\left\|u^{\prime}\right\|_{H^{2}(\Omega)}+\|\bar{u}\|_{H^{2}\left(\Omega_{x}\right)}+\left\|u^{\prime}\left(\cdot, z_{\iota}\right)\right\|_{H^{1}\left(\Omega_{x}\right)} \leq C_{f, g_{ \pm}}\left(1+\varepsilon_{M}\right),
$$

with some constant $C_{f, g_{ \pm}}>0$ independent of $\varepsilon$, however dependent on the source terms $f$ respectively $g_{ \pm}$. Recall that $\bar{u}$ is the average of $u$ over $z$ and $u^{\prime}:=u-\bar{u}$.

Proof: The proof of this lemma uses standard "energy"-estimates and density arguments as well as the Poincaré and trace-theorems. See [3, 10, 12, for more details.

Standard elliptic results permit to show that, under the additional hypothesis of this theorem, the solution of (2.4) is more regular, i.e. $u \in H^{2}(\Omega)$.

Remark then that multiplying (2.4) by $u$ and integrating over $\Omega$ yields by integration by parts the $H^{1}$-estimate

$$
\left\|\partial_{x} u\right\|_{L^{2}}^{2}+\left\|\frac{1}{\sqrt{\varepsilon(z)}} \partial_{z} u\right\|_{L^{2}}^{2} \leq C\left(\|f\|_{L^{2}}^{2}+\left\|g_{+}\right\|_{L_{x}^{2}}^{2}+\left\|g_{-}\right\|_{L_{x}^{2}}^{2}\right) .
$$

Rewriting now the equation as

$$
-A_{x} \partial_{x x} u-\partial_{z}\left(\frac{A_{z}}{\varepsilon(z)} \partial_{z} u\right)=f+\left(\partial_{x} A_{x}\right) \partial_{x} u,
$$


multiplying it by $-\partial_{x x} u$ and integrating over $\Omega$ yields (by integration by parts)

$$
\begin{aligned}
& \left\|\sqrt{A_{x}} \partial_{x x} u\right\|^{2}+\left\|\sqrt{\frac{A_{z}}{\varepsilon(z)}} \partial_{x z} u\right\|^{2}+\int_{\Omega} \frac{\partial_{x} A_{z}}{\varepsilon(z)} \partial_{z} u \partial_{x z} u d x d z \\
& \quad \leq\|f\|\left\|\partial_{x x} u\right\|+C\left\|\partial_{x} u\right\|\left\|\partial_{x x} u\right\|+\int_{\Omega_{x}} \partial_{x} g_{+} \partial_{x} u\left(x, z_{+}\right) d x-\int_{\Omega_{x}} \partial_{x} g_{-} \partial_{x} u\left(x, z_{-}\right) d x
\end{aligned}
$$

Using now the $H^{1}$-estimate (3.19) and the fact that $\left\|\partial_{x} u\left(x, z_{ \pm}\right)\right\|_{L_{x}^{2}} \leq C\left\|\partial_{x} u\right\|_{L^{2}}+$ $C\left\|\partial_{x z} u\right\|_{L^{2}}$, yields the first $H^{2}$-estimates

$$
\left\|\partial_{x x} u\right\|_{L^{2}}^{2}+\left\|\frac{1}{\sqrt{\varepsilon(z)}} \partial_{x z} u\right\|_{L^{2}}^{2} \leq C_{f, g_{ \pm}}\left(1+\varepsilon_{\max }\right) .
$$

Coming now back to the equation

$$
-\partial_{z}\left(\frac{A_{z}}{\varepsilon(z)} \partial_{z} u\right)=f+\partial_{x}\left(A_{x} \partial_{x} u\right)
$$

one gets with (3.21)

$$
\left\|\partial_{z}\left(\frac{A_{z}}{\varepsilon(z)} \partial_{z} u\right)\right\| \leq C_{f, g_{ \pm}}\left(1+\varepsilon_{\max }\right)
$$

Hence, remarking that

$$
\frac{A_{z}}{\varepsilon(z)} \partial_{z} u(x, z)=\frac{A_{z}}{\varepsilon\left(z_{-}\right)} \partial_{z} u\left(x, z_{-}\right)+\int_{z_{-}}^{z} \partial_{z}\left(\frac{A_{z}}{\varepsilon(\tau)} \partial_{z} u(x, \tau)\right) d \tau
$$

permits to show an improved $H^{1}$-estimate

$$
\left\|\frac{1}{\varepsilon(z)} \partial_{z} u\right\|_{L^{2}} \leq C\left\|g_{-}\right\|_{L_{x}^{2}}+C\left\|\partial_{z}\left(\frac{A_{z}}{\varepsilon(z)} \partial_{z} u\right)\right\|_{L^{2}} \leq C_{f, g_{ \pm}}\left(1+\varepsilon_{\max }\right) .
$$

Finally, with all these estimates, one has

$$
\partial_{z z} u=\partial_{z}\left(\frac{\varepsilon(z)}{A_{z}} \frac{A_{z}}{\varepsilon(z)} \partial_{z} u\right)=\partial_{z}\left(\frac{\varepsilon(z)}{A_{z}}\right) \frac{A_{z}}{\varepsilon(z)} \partial_{z} u+\frac{\varepsilon(z)}{A_{z}} \partial_{z}\left(\frac{A_{z}}{\varepsilon(z)} \partial_{z} u\right),
$$

yielding

$$
\left\|\partial_{z z} u\right\|_{L^{2}} \leq\left\|\partial_{z}\left(\frac{\varepsilon(z)}{A_{z}}\right)\right\|_{L^{\infty}}\left\|\frac{A_{z}}{\varepsilon(z)} \partial_{z} u\right\|_{L^{2}}+C \varepsilon_{\max }\left\|\partial_{z}\left(\frac{A_{z}}{\varepsilon(z)} \partial_{z} u\right)\right\|_{L^{2}} \leq C_{f, g_{ \pm}} \varepsilon_{M}
$$

which concludes the proof together with (3.21).

From now on, we shall not carry with us the bound of $\|\varepsilon\|_{W^{1, \infty}\left(\Omega_{z}\right)}$, i.e. $\varepsilon_{M}$, keeping in mind that the anisotropy has to be bounded from above in $W^{1, \infty}$. 
Theorem 3.2 Under Hypothesis $B$, the function $\xi_{2}^{\prime} \in \mathcal{V}_{2}$, part of the solution of the $(A P / A P)$-model (3.13), converges towards zero in $H^{1}\left(\Omega_{2}\right)$ as $\varepsilon\left(z_{\iota}\right) \rightarrow 0$. If we assume moreover that $u_{1}^{\prime}\left(\cdot, z_{\iota}\right) \in H^{2}\left(\Omega_{x}\right)$, then there exists a constant $c>0$, independent of $\varepsilon\left(z_{\iota}\right)$, such that

$$
\left\|\partial_{x} \xi_{2}^{\prime}\right\|_{L^{2}\left(\Omega_{2}\right)} \leq c \sqrt{\varepsilon\left(z_{\iota}\right)} \quad \text { and } \quad\left\|\partial_{z} \xi_{2}^{\prime}\right\|_{L^{2}\left(\Omega_{2}\right)} \leq c \varepsilon\left(z_{\iota}\right)
$$

Proof: The proof of this theorem is very similar to the proof of the previous lemma. Let us only recall that $\xi_{2}^{\prime}$ is the solution of a diffusion problem with slightly different boundary condition on one side, when compared with the P-problem, i.e.

$$
\begin{cases}-\partial_{x}\left(A_{x} \partial_{x} \xi_{2}^{\prime}\right)-\partial_{z}\left(\frac{A_{z}}{\varepsilon(z)} \partial_{z} \xi_{2}^{\prime}\right)=h & \text { for }(x, z) \in \Omega_{x} \times \Omega_{z}^{2} \\ \frac{A_{z}\left(x, z_{-}\right)}{\varepsilon\left(z_{-}\right)} \partial_{z} \xi_{2}^{\prime}\left(x, z_{-}\right)=g_{-}(x), & \text { for } x \in \Omega_{x}, \\ \xi_{2}^{\prime}\left(x, z_{\iota}\right)=0, & \text { for } x \in \Omega_{x} \\ \xi_{2}^{\prime}\left(x_{ \pm}, z\right)=0, & \text { for } z \in \Omega_{z}^{2},\end{cases}
$$

where we denoted for simplicity reasons $h:=f+\partial_{x}\left(A_{x} \partial_{x} u_{1}^{\prime}\left(x, z_{\iota}\right)\right)+\partial_{x}\left(A_{x} \partial_{x} \bar{u}\right)$. Let us first assume that $u_{1}^{\prime}\left(\cdot, z_{\iota}\right) \in H^{2}\left(\Omega_{x}\right)$, such that $h \in L^{2}\left(\Omega_{2}\right)$. Multiplying now the system (3.25) by $\xi_{2}^{\prime}$ and integrating over $\Omega_{x} \times \Omega_{z}^{2}$ yields

$$
\left\|\sqrt{A_{x}} \partial_{x} \xi_{2}^{\prime}\right\|_{L_{2}^{2}}^{2}+\left\|\sqrt{\frac{A_{z}}{\varepsilon(z)}} \partial_{z} \xi_{2}^{\prime}\right\|_{L_{2}^{2}}^{2}=\int_{\Omega^{2}} h \xi_{2}^{\prime} \mathrm{d} x \mathrm{~d} z-\int_{\Omega_{x}} g_{-}(x) \xi_{2}^{\prime}\left(x, z_{-}\right) \mathrm{d} x .
$$

Remarking that $\frac{1}{\varepsilon(z)} \geq \frac{1}{\varepsilon\left(z_{\iota}\right)}$ in $\Omega_{z}^{2}$, and that $\xi_{2}^{\prime}\left(x, z_{\iota}\right)=0$ for all $x \in \Omega_{x}$, such that by Poincaré's inequality

$$
\left\|\xi_{2}^{\prime}\left(\cdot, z_{-}\right)\right\|_{L^{2}\left(\Omega_{x}\right)} \leq c\left\|\partial_{z} \xi_{2}^{\prime}\right\|_{L^{2}\left(\Omega_{2}\right)} \quad \text { and } \quad\left\|\xi_{2}^{\prime}\right\|_{L^{2}\left(\Omega_{2}\right)} \leq c\left\|\partial_{z} \xi_{2}^{\prime}\right\|_{L^{2}\left(\Omega_{2}\right)},
$$

we obtain by Cauchy-Schwarz

$$
\left\|\partial_{x} \xi_{2}^{\prime}\right\|_{L^{2}\left(\Omega_{2}\right)}^{2}+\frac{1}{\varepsilon\left(z_{\iota}\right)}\left\|\partial_{z} \xi_{2}^{\prime}\right\|_{L^{2}\left(\Omega_{2}\right)}^{2} \leq c\left[\|h\|_{L^{2}\left(\Omega_{2}\right)}+\left\|g_{-}\right\|_{L^{2}\left(\Omega_{x}\right)}\right]\left\|\partial_{z} \xi_{2}^{\prime}\right\|_{L^{2}\left(\Omega_{2}\right)},
$$

leading to

$$
\varepsilon\left(z_{\iota}\right)\left\|\partial_{x} \xi_{2}^{\prime}\right\|_{L^{2}\left(\Omega_{2}\right)}^{2}+\left\|\partial_{z} \xi_{2}^{\prime}\right\|_{L^{2}\left(\Omega_{2}\right)}^{2} \leq c\left(h, g_{-}\right) \varepsilon\left(z_{\iota}\right)^{2},
$$

which completes the proof in the regular case. Remark that it is at this point that our proof differs from the $H^{1}$-estimates (3.19), (3.22) of the previous proof, and this thanks to the Poincaré inequality (3.26), valid due to the boundary condition $\xi_{2}^{\prime}\left(x, z_{\iota}\right)=0$, $\forall x \in \Omega_{x}$. It is exactly this condition which implies the convergence of $\xi_{2}^{\prime}$ towards zero.

Let us suppose now that we have only $u_{1}^{\prime}\left(\cdot, z_{\iota}\right) \in H^{1}\left(\Omega_{x}\right)$, which is immediate under Hypothesis $\mathrm{B}$, such that $h$ belongs only to $H^{-1}\left(\Omega_{2}\right)$. In this case, following the same arguments as above, would lead rather to

$$
\left\|\partial_{x} \xi_{2}^{\prime}\right\|_{L^{2}\left(\Omega_{2}\right)}^{2}+\frac{1}{\varepsilon\left(z_{\iota}\right)}\left\|\partial_{z} \xi_{2}^{\prime}\right\|_{L^{2}\left(\Omega_{2}\right)}^{2} \leq c\|h\|_{H^{-1}\left(\Omega_{2}\right)}\left\|\xi_{2}^{\prime}\right\|_{H^{1}\left(\Omega_{2}\right)},+c\left\|g_{-}\right\|_{L^{2}\left(\Omega_{x}\right)}\left\|\partial_{z} \xi_{2}^{\prime}\right\|_{L^{2}\left(\Omega_{2}\right)}
$$


leading only to

$$
\left\|\partial_{x} \xi_{2}^{\prime}\right\|_{L^{2}\left(\Omega_{2}\right)}^{2}+\frac{1}{\varepsilon\left(z_{\iota}\right)}\left\|\partial_{z} \xi_{2}^{\prime}\right\|_{L^{2}\left(\Omega_{2}\right)}^{2} \leq c\left(\|h\|_{H^{-1}\left(\Omega_{2}\right)}^{2}+\sqrt{\varepsilon\left(z_{\iota}\right)}\left\|g_{-}\right\|_{L^{2}\left(\Omega_{x}\right)}^{2}\right)
$$

which does not permit to have the desired convergence. However, density arguments permit to conclude the proof in the general case. Indeed, let us consider the linear, continuous mapping

$$
\mathcal{U}: h \in H^{-1}\left(\Omega_{2}\right) \longmapsto \xi_{2}^{\prime} \in H^{1}\left(\Omega_{2}\right) \text { sol. of (3.25). }
$$

Let now $h \in H^{-1}\left(\Omega_{2}\right)$. Then for each $\delta>0$ there exists by density a more regular data $\tilde{h} \in L^{2}\left(\Omega_{2}\right)$ such that $\|h-\tilde{h}\|_{H^{-1}\left(\Omega_{2}\right)} \leq \delta$ and $\|\mathcal{U}(\tilde{h})\|_{H^{1}\left(\Omega_{2}\right)} \leq c(\tilde{h}) \sqrt{\varepsilon\left(z_{\iota}\right)}$ by the estimate (3.27). As the map $\mathcal{U}$ is continuous and linear, one has then

$$
\begin{aligned}
\|\mathcal{U}(h)\|_{H^{1}\left(\Omega_{2}\right)} \leq\|\mathcal{U}(h-\tilde{h})\|_{H^{1}\left(\Omega_{2}\right)}+\|\mathcal{U}(\tilde{h})\|_{H^{1}\left(\Omega_{2}\right)} & \leq c\|h-\tilde{h}\|_{H^{-1}\left(\Omega_{2}\right)}+c(\tilde{h}) \sqrt{\varepsilon\left(z_{\iota}\right)} \\
& \leq c \delta+c(\tilde{h}) \sqrt{\varepsilon\left(z_{\iota}\right)} .
\end{aligned}
$$

Thus, for all $\tau>0$, one can find $\delta>0$ and $\varepsilon\left(z_{\iota}\right)>0$ such that $\|\mathcal{U}(h)\|_{H^{1}\left(\Omega_{2}\right)} \leq \tau$ for all $\varepsilon<\varepsilon\left(z_{\iota}\right)$, which proves the convergence of $\mathcal{U}(h)$ towards zero in $H^{1}\left(\Omega_{2}\right)$, as $\varepsilon\left(z_{\iota}\right) \rightarrow 0$.

Let us now come to the study of the error on the mean part $\bar{u}-\bar{v}$ and the fluctuation part $u_{1}^{\prime}-v_{1}^{\prime}$, when approximating the (AP/AP)-model with the (AP/L)-model.

Theorem 3.3 Let $\left(\bar{u}, u_{1}^{\prime}, \xi_{2}^{\prime}\right) \in \mathcal{W} \times \mathcal{V}_{1} \times \mathcal{V}_{2}$ be the solution of (3.13) and $\left(\bar{v}, v_{1}^{\prime}, 0\right) \in$ $\mathcal{W} \times \mathcal{V}_{1} \times \mathcal{V}_{2}$ the solution of (3.14) and let us assume Hypothesis $B$ to be satisfied. Then one has

$$
\bar{u} \rightarrow_{\varepsilon\left(z_{\iota}\right) \rightarrow 0} \bar{v} \text { in } \mathcal{W} \text { and } u_{1}^{\prime} \rightarrow_{\varepsilon\left(z_{\iota}\right) \rightarrow 0} v_{1}^{\prime} \text { in } \mathcal{V}_{1}
$$

If we suppose moreover that $u_{1}^{\prime}\left(\cdot, z_{\iota}\right) \in H^{2}\left(\Omega_{x}\right)$, then there exists a positive constant $c$, independent of $\varepsilon\left(z_{\iota}\right)$, such that

$$
\left\|\partial_{x}(\bar{u}-\bar{v})\right\|_{L^{2}\left(\Omega_{x}\right)}+\left\|\partial_{x}\left(u_{1}^{\prime}-v_{1}^{\prime}\right)\right\|_{L^{2}\left(\Omega_{1}\right)}+\left\|\partial_{z}\left(u_{1}^{\prime}-v_{1}^{\prime}\right)\right\|_{L^{2}\left(\Omega_{1}\right)} \leq c \sqrt{\varepsilon\left(z_{\iota}\right)} .
$$

Proof: To simplify the notations, we shall denote in this proof the difference between the two solutions of (3.13) resp. (3.14) by $\left(\bar{w}, w_{1}^{\prime}, \xi_{2}^{\prime}\right)$, where $\bar{w}:=\bar{u}-\bar{v} \in \mathcal{W}$ and $w_{1}^{\prime}:=u_{1}^{\prime}-v_{1}^{\prime} \in \mathcal{V}_{1}$. Aim is to show that both $\bar{w}$ and $w_{1}^{\prime}$ converge towards zero in the respective spaces. This shall be done in several steps.

First, let us suppose that $u_{1}^{\prime}\left(\cdot, z_{\iota}\right) \in H^{2}\left(\Omega_{x}\right)$ and start by writing the system of equations satisfied by $\bar{w}$ and $w_{1}^{\prime}$ :

$$
\left\{\begin{array}{l}
-\partial_{x}\left(\overline{A_{x}} \partial_{x} \bar{w}\right)=\frac{1}{L_{z}} \partial_{x}\left(\int_{\Omega_{z}^{1}} A_{x}^{\prime} \partial_{x} w_{1}^{\prime} \mathrm{d} z\right. \\
\left.\quad+\int_{\Omega_{z}^{2}} A_{x}^{\prime} \partial_{x} w_{1}^{\prime}\left(x, z_{\iota}\right) \mathrm{d} z+\int_{\Omega_{z}^{2}} A_{x}^{\prime} \partial_{x} \xi_{2}^{\prime} \mathrm{d} z\right), \quad \text { for } x \in \Omega_{x} \\
\bar{w}\left(x_{ \pm}\right)=0
\end{array}\right.
$$




$$
\begin{cases}-\partial_{x}\left(A_{x} \partial_{x} w_{1}^{\prime}\right)-\partial_{z}\left(\frac{A_{z}}{\varepsilon(z)} \partial_{z} w_{1}^{\prime}\right)=\partial_{x}\left(A_{x} \partial_{x} \bar{w}\right), & \text { for }(x, z) \in \Omega_{x} \times \Omega_{z}^{1}, \\ \frac{A_{z}\left(x, z_{+}\right)}{\varepsilon\left(z_{+}\right)} \partial_{z} w_{1}^{\prime}\left(x, z_{+}\right)=0, & \text { for } x \in \Omega_{x}, \\ w_{1}^{\prime}\left(x_{ \pm}, z\right)=0, & \text { for } z \in \Omega_{z}^{1}, \\ \frac{A_{z}\left(x, z_{\iota}\right)}{\varepsilon\left(z_{\iota}\right)} \partial_{z} w_{1}^{\prime}\left(x, z_{\iota}\right)=\frac{A_{z}\left(x, z_{\iota}\right)}{\varepsilon\left(z_{\iota}\right)} \partial_{z} \xi_{2}^{\prime}\left(x, z_{\iota}\right), & \text { for } x \in \Omega_{x} \\ \int_{\Omega_{z}^{1}} w_{1}^{\prime}(x, z) \mathrm{d} z+L_{z}^{2} w_{1}^{\prime}\left(x, z_{\iota}\right)+\int_{\Omega_{z}^{2}} \xi_{2}^{\prime}(x, z) \mathrm{d} z=0, & \text { for } x \in \Omega_{x} \text { (constraint). }\end{cases}
$$

Introducing on $\Omega_{x} \times \Omega_{z}^{1}$ the variable $w(x, z):=\bar{w}(x)+w_{1}^{\prime}(x, z)$, this one solves:

$$
\begin{cases}-\partial_{x}\left(A_{x} \partial_{x} w\right)-\partial_{z}\left(\frac{A_{z}}{\varepsilon(z)} \partial_{z} w\right)=0, & \text { for }(x, z) \in \Omega_{x} \times \Omega_{z}^{1}, \\ \frac{A_{z}\left(x, z_{+}\right)}{\varepsilon\left(z_{+}\right)} \partial_{z} w\left(x, z_{+}\right)=0, & \text { for } x \in \Omega_{x}, \\ w\left(x_{ \pm}, z\right)=0, & \text { for } z \in \Omega_{z}^{1}, \\ \frac{A_{z}\left(x, z_{\iota}\right)}{\varepsilon\left(z_{\iota}\right)} \partial_{z} w\left(x, z_{\iota}\right)=\frac{A_{z}\left(x, z_{\iota}\right)}{\varepsilon\left(z_{\iota}\right)} \partial_{z} \xi_{2}^{\prime}\left(x, z_{\iota}\right), & \text { for } x \in \Omega_{x} .\end{cases}
$$

1st Step: $H^{1}$-estimate of $w$ :

Multiplying (3.33) by $w$ and integrating on $\Omega_{x} \times \Omega_{z}^{1}$ by parts, yields

$$
\left\|\sqrt{A_{x}} \partial_{x} w\right\|_{L_{1}^{2}}^{2}+\left\|\sqrt{\frac{A_{z}}{\varepsilon(z)}} \partial_{z} w\right\|_{L_{1}^{2}}^{2}+\int_{\Omega_{x}} \frac{A_{z}\left(x, z_{\iota}\right)}{\varepsilon\left(z_{\iota}\right)} \partial_{z} \xi_{2}^{\prime}\left(x, z_{\iota}\right) w\left(x, z_{\iota}\right) \mathrm{d} x=0 .
$$

The difficulty in estimating $w$ comes now from the term $\int_{\Omega_{x}} \frac{A_{z}\left(x, z_{\iota}\right)}{\varepsilon\left(z_{\iota}\right)} \partial_{z} \xi_{2}^{\prime}\left(x, z_{\iota}\right) w\left(x, z_{\iota}\right) \mathrm{d} x$. To be able to reformulate this term, we shall come back to (3.31)-(3.32).

The constraint in (3.32) can be reformulated as

$$
\begin{array}{r}
0=\partial_{x}\left(\overline{A_{x}} \partial_{x}\left[\int_{\Omega_{z}^{1}} w_{1}^{\prime} \mathrm{d} z+\int_{\Omega_{z}^{2}} w_{1}^{\prime}\left(x, z_{\iota}\right) \mathrm{d} z+\int_{\Omega_{z}^{2}} \xi_{2}^{\prime} \mathrm{d} z\right]\right) \\
=\partial_{x}\left(\int_{\Omega_{z}^{1}} \overline{A_{x}} \partial_{x} w_{1}^{\prime} \mathrm{d} z+\int_{\Omega_{z}^{2}} \overline{A_{x}} \partial_{x} w_{1}^{\prime}\left(x, z_{\iota}\right) \mathrm{d} z+\int_{\Omega_{z}^{2}} \overline{A_{x}} \partial_{x} \xi_{2}^{\prime} \mathrm{d} z\right),
\end{array}
$$

which allows to rewrite (3.31) as

$$
-\partial_{x}\left(\overline{A_{x}} \partial_{x} \bar{w}\right)=\frac{1}{L_{z}} \partial_{x}\left(\int_{\Omega_{z}^{1}} A_{x} \partial_{x} w_{1}^{\prime} \mathrm{d} z+\int_{\Omega_{z}^{2}} A_{x} \partial_{x} w_{1}^{\prime}\left(x, z_{\iota}\right) \mathrm{d} z+\int_{\Omega_{z}^{2}} A_{x} \partial_{x} \xi_{2}^{\prime} \mathrm{d} z\right) .
$$

Integrating now (3.32) on $\Omega_{z}^{1}$ gives

$$
-\int_{\Omega_{z}^{1}} \partial_{x}\left(A_{x} \partial_{x} w_{1}^{\prime}\right) \mathrm{d} z+\frac{A_{z}\left(x, z_{\iota}\right)}{\varepsilon\left(z_{\iota}\right)} \partial_{z} \xi_{2}^{\prime}\left(x, z_{\iota}\right)=\int_{\Omega_{z}^{1}} \partial_{x}\left(A_{x} \partial_{x} \bar{w}\right) \mathrm{d} z
$$


and (3.35) becomes then

$$
\begin{aligned}
-\frac{1}{L_{z}} \int_{\Omega_{z}} \partial_{x}\left(A_{x} \partial_{x} \bar{w}\right) \mathrm{d} z & =\frac{1}{L_{z}}\left(-\int_{\Omega_{z}^{1}} \partial_{x}\left(A_{x} \partial_{x} \bar{w}\right) \mathrm{d} z+\frac{A_{z}\left(x, z_{\iota}\right)}{\varepsilon\left(z_{\iota}\right)} \partial_{z} \xi_{2}^{\prime}\left(x, z_{\iota}\right)\right. \\
& \left.+\int_{\Omega_{z}^{2}} \partial_{x}\left(A_{x} \partial_{x} w_{1}^{\prime}\left(x, z_{\iota}\right)\right) \mathrm{d} z+\int_{\Omega_{z}^{2}} \partial_{x}\left(A_{x} \partial_{x} \xi_{2}^{\prime}\right) \mathrm{d} z\right),
\end{aligned}
$$

which is finally equivalent to

$$
\frac{A_{z}\left(x, z_{\iota}\right)}{\varepsilon\left(z_{\iota}\right)} \partial_{z} \xi_{2}^{\prime}\left(x, z_{\iota}\right)=-\int_{\Omega_{z}^{2}} \partial_{x}\left(A_{x} \partial_{x} w\left(x, z_{\iota}\right)\right) \mathrm{d} z-\int_{\Omega_{z}^{2}} \partial_{x}\left(A_{x} \partial_{x} \xi_{2}^{\prime}\right) \mathrm{d} z .
$$

Using this last equation in (3.34), permits to get

$$
\left\|\sqrt{A_{x}} \partial_{x} w\right\|_{L_{1}^{2}}^{2}+\left\|\sqrt{\frac{A_{z}}{\varepsilon(z)}} \partial_{z} w\right\|_{L_{1}^{2}}^{2}-\left(\partial_{x}\left(A_{x} \partial_{x} w\left(\cdot, z_{\iota}\right)\right)-\partial_{x}\left(A_{x} \partial_{x} \xi_{2}^{\prime}\right), w\left(\cdot, z_{\iota}\right)\right)_{L_{2}^{2}}=0 .
$$

Integrating by parts in the last two terms, yields

$$
\left\|\sqrt{A_{x}} \partial_{x} w\right\|_{L_{1}^{2}}^{2}+\left\|\sqrt{\frac{A_{z}}{\varepsilon(z)}} \partial_{z} w\right\|_{L_{1}^{2}}^{2}+\left\|\sqrt{A_{x}} \partial_{x} w\left(\cdot, z_{\iota}\right)\right\|_{L_{2}^{2}}^{2}=-\left(A_{x} \partial_{x} \xi_{2}^{\prime}, \partial_{x} w\left(\cdot, z_{\iota}\right)\right)_{L_{2}^{2}},
$$

which permits to obtain, as $\frac{1}{\varepsilon(z)} \geq \frac{1}{\varepsilon_{\max }}$ in $\Omega_{1}$,

$$
\left\|\partial_{x} w\right\|_{L^{2}\left(\Omega_{1}\right)}^{2}+\left\|\partial_{z} w\right\|_{L^{2}\left(\Omega_{1}\right)}^{2}+\left\|\partial_{x} w\left(\cdot, z_{\iota}\right)\right\|_{L^{2}\left(\Omega_{x}\right)}^{2} \leq c\left\|\partial_{x} \xi_{2}^{\prime}\right\|_{L^{2}\left(\Omega_{2}\right)}\left\|\partial_{x} w\left(\cdot, z_{\iota}\right)\right\|_{L^{2}\left(\Omega_{x}\right)},
$$

leading thanks to Theorem 3.2 to the $H^{1}$-estimate of $w$

$$
\left\|\partial_{x} w\right\|_{L^{2}\left(\Omega_{1}\right)}^{2}+\left\|\partial_{z} w\right\|_{L^{2}\left(\Omega_{1}\right)}^{2}+\left\|\partial_{x} w\left(\cdot, z_{\iota}\right)\right\|_{L^{2}\left(\Omega_{x}\right)}^{2} \leq c\left\|\partial_{x} \xi_{2}^{\prime}\right\|_{L^{2}\left(\Omega_{2}\right)}^{2} \leq c \varepsilon\left(z_{\iota}\right) .
$$

2nd Step: $H^{1}$-estimate of $\bar{w}$ and $w_{1}^{\prime}$ :

To estimate from this last inequality the functions $\bar{w}$ and $w_{1}^{\prime}$, we shall use the constraint. Indeed, one has

$$
\int_{\Omega_{z}^{1}} w(x, z)(x, z) \mathrm{d} z+L_{z}^{2} w\left(x, z_{\iota}\right)+\int_{\Omega_{z}^{2}} \xi_{2}^{\prime}(x, z) \mathrm{d} z=L_{z} \bar{w}(x), \quad \forall x \in \Omega_{x},
$$

implying

$$
\|\bar{w}\|_{L^{2}\left(\Omega_{x}\right)} \leq c\left(\|w\|_{L^{2}\left(\Omega_{1}\right)}+\left\|w\left(\cdot, z_{\iota}\right)\right\|_{L^{2}\left(\Omega_{x}\right)}+\left\|\xi_{2}^{\prime}\right\|_{L^{2}\left(\Omega_{2}\right)}\right) \leq c \sqrt{\varepsilon\left(z_{\iota}\right)} .
$$

As furthermore $w_{1}^{\prime}=w-\bar{w}$, we can conclude the proof in the regular case. In the less regular case, Theorem 3.2 as well as formulæ (3.42), (3.43) permit to get the convergences in $H^{1}$.

Estimates (3.24) and (3.30) will permit in the following to measure the error done by using the computationally more advantageous (AP/L)-hybrid model instead of a full AP-model for the resolution of (2.4), and to place the interface-position $z_{\iota}$. 


\section{Numerical discretization and investigations of the hybrid model}

The aim of this section is the discretization of the (AP/L)-coupling model (3.17) by means of a finite element method, and the discussion of the obtained numerical results.

\subsection{Finite element discretization}

Let us present here in some details the finite element method we used.

The computational domain $\Omega_{x} \times \Omega_{z}$ with $\Omega_{x}=\left[x_{-}, x_{+}\right]$and $\Omega_{z}=\left[z_{-}, z_{+}\right]$is decomposed into a set of rectangular cells $\left[x_{i}, x_{i+1}\right] \times\left[z_{k}, z_{k+1}\right]$ where

$$
\begin{array}{lll}
x_{i}=x_{-}+i \Delta x, & i=0, \ldots, N_{x}+1, & \Delta x=\left(x_{+}-x_{-}\right) /\left(N_{x}+1\right), \\
z_{k}=z_{-}+k \Delta z, & k=0, \ldots, N_{z}+1, & \Delta z=\left(z_{+}-z_{-}\right) /\left(N_{z}+1\right) .
\end{array}
$$

The domain $\Omega_{z}$ is decomposed into $\Omega_{z}^{1}$ and $\Omega_{z}^{2}, z_{\iota}$ with $\iota \in\left\{1, \ldots, N_{z}\right\}$ denoting the interface delimiting these two sub-domains so that $\Omega_{z}^{1}$ is composed of the cells $\left[z_{k}, z_{k+1}\right]$ for $k=\iota, \ldots, N_{z}$.

The functional spaces $\mathcal{V}$ resp. $\mathcal{V}_{1}$ are approximated by $\mathbb{Q}_{1}$ finite element spaces, denoted $\mathcal{V}_{h}$ resp. $\mathcal{V}_{1, h}$. The mean functions and the Lagrangian belonging to $\mathcal{W}$ and $\mathcal{L}$ are approximated by a $\mathbb{P}_{1}$ finite element giving rise to the definition of $\mathcal{W}_{h}$ and $\mathcal{L}_{h}$. In the sequel, $\chi_{i}(x)$ and $\kappa_{k}(z)$ denote the standard $\mathbb{P}_{1}$ hat functions respectively centered in $x_{i}$ and $z_{k}$.

The weak formulation of the hybrid (AP/L)-model (3.17) is approximated thanks to a three points Gauss quadrature formula, yielding the following linear system

$$
(A P / L)_{h} \quad\left(\begin{array}{ccc}
A_{x a} & \frac{1}{L_{z}}\left(C_{a 1}+C_{a 2}^{\iota}\right) & 0 \\
C_{f 1} & A_{x f 1}+A_{z 1} & B_{l 1} \\
0 & B_{c 1}+B_{c 2}^{\iota} & 0
\end{array}\right)\left(\begin{array}{c}
\alpha \\
\beta \\
\gamma
\end{array}\right)=\left(\begin{array}{c}
F_{\bar{u}} \\
F_{u_{1}^{\prime}} \\
0
\end{array}\right)
$$

where $A_{\star}$ (resp. $B_{\star}, C_{\star}$ ) is the matrix associated to the bilinear form $a_{\star}$ (resp. $b_{\star}, c_{\star}$ ) introduced in (3.15). Note that the matrices $C_{a 2}, B_{c 2} \in \mathbb{R}^{N_{x} \times N_{x}}$ associated to $a_{a 2}, b_{c 2}$ involve only the nodes located on the interface, such that they are expanded to $C_{a 2}^{\iota}, B_{c 2}^{\iota}$ by adding zero elements in order to conform with the size of $C_{a 1}, B_{c 1} \in \mathbb{R}^{N_{x} \times N_{x}\left(N_{z}+2-\iota\right)}$ to define the system (4.44). The right-hand side definition is specified here

$$
\begin{aligned}
\left(F_{\bar{u}}\right)_{i} & :=\left(\bar{f}, \chi_{i}\right)_{L^{2}\left(\Omega_{x}\right)}+\frac{1}{L_{z}}\left(g_{+}-g_{-}, \chi_{i}\right)_{L^{2}\left(\Omega_{x}\right)}, \forall i=1, \ldots, N_{x}, \\
\left(F_{u_{1}^{\prime}}\right)_{i k} & :=\left(f, \chi_{i} \kappa_{k}\right)_{L^{2}\left(\Omega_{1}\right)}+\left(g_{+}, \chi_{i} \kappa_{k}\left(z_{+}\right)\right)_{L^{2}\left(\Omega_{x}\right)}, \forall i=1, \ldots, N_{x}, k=\iota, \ldots, N_{z}+1,
\end{aligned}
$$

and the unknowns of the system are three vectors $\alpha \in \mathbb{R}^{N_{x}}, \beta \in \mathbb{R}^{N_{x}\left(N_{z}+2-\iota\right)}$ and $\gamma \in \mathbb{R}^{N_{x}+2}$

$$
\begin{aligned}
\alpha & :=\left(\alpha_{1} \cdots \alpha_{N_{x}}\right)^{T}, \quad \beta:=\left(\beta_{1 \iota} \cdots \beta_{1 N_{z}+1} \cdots \beta_{N_{x} \iota} \cdots \beta_{N_{x} N_{z}+1}\right)^{T} \\
\gamma & :=\left(\gamma_{0} \cdots \gamma_{N_{x}+1}\right)^{T} .
\end{aligned}
$$


As a comparison, the linear system corresponding to the AP-model (2.10) writes

$$
(A P)_{h} \quad\left(\begin{array}{ccc}
A_{x a} & \frac{1}{L_{z}} C_{a} & 0 \\
C_{f} & A_{x f}+A_{z} & B_{l} \\
0 & B_{c} & 0
\end{array}\right)\left(\begin{array}{c}
\alpha \\
\tilde{\beta} \\
\gamma
\end{array}\right)=\left(\begin{array}{c}
F_{\bar{u}} \\
F_{u^{\prime}} \\
0
\end{array}\right)
$$

where $A_{z}$ (resp. $A_{x f}, B_{l}, B_{c}, C_{a}$ and $C_{f}$ ) is associated to the bilinear form similar to $a_{z 1}$ (resp. $a_{x f 1}, b_{l 1}, b_{c 1}, c_{a 1}$ and $c_{f 1}$ ) but integrated on $\Omega_{z}$ instead of $\Omega_{z}^{1}$. The right-hand side $F_{u^{\prime}}$ is defined for $i=1, \ldots, N_{x}, k=0, \ldots, N_{z}+1$, by

$$
\left(F_{u^{\prime}}\right)_{i k}:=\left(f, \chi_{i} \kappa_{k}\right)_{L^{2}(\Omega)}+\left(g_{+}, \chi_{i} \kappa_{k}\left(z_{+}\right)\right)_{L^{2}\left(\Omega_{x}\right)}-\left(g, \chi_{i} \kappa_{k}\left(z_{-}\right)\right)_{L^{2}\left(\Omega_{x}\right)},
$$

and the unknown $\tilde{\beta} \in \mathbb{R}^{N_{x}\left(N_{z}+2\right)}$ writes

$$
\tilde{\beta}:=\left(\beta_{10} \cdots \beta_{1 N_{z}+1} \cdots \beta_{N_{x} 0} \cdots \beta_{N_{x} N_{z}+1}\right)^{T} .
$$

The gain of the hybrid (AP/L)-model as compared to the fully AP-model results from the size reduction of the fluctuation unknown. Indeed, $\tilde{\beta}$ is a $N_{x}\left(N_{z}+2\right)$-vector, while $\beta$ is a $N_{x}\left(N_{z}+2-\iota\right)$-vector.

As for the P-model (2.5), the corresponding linear system is of the form

$$
(P)_{h} \quad\left(A_{x f}+A_{z}\right) \delta=F,
$$

where the second member is defined for $i=1, \ldots, N_{x}, k=0, \ldots, N_{z}+1$, by

$$
(F)_{i k}:=\left(f, \chi_{i} \kappa_{k}\right)_{L^{2}(\Omega)}+\left(g_{+}, \chi_{i} \kappa_{k}\left(z_{+}\right)\right)_{L^{2}\left(\Omega_{x}\right)}-\left(g, \chi_{i} \kappa_{k}\left(z_{-}\right)\right)_{L^{2}\left(\Omega_{x}\right)},
$$

and the unknown $\delta \in \mathbb{R}^{N_{x}\left(N_{z}+2\right)}$ writes

$$
\delta:=\left(\delta_{10} \cdots \delta_{1 N_{z}+1} \cdots \delta_{N_{x} 0} \cdots \delta_{N_{x} N_{z}+1}\right)^{T} .
$$

Note that the linear system providing the fluctuation in (4.45) is nothing but the P-model matrix (4.46) augmented with the two sub-matrices $B_{l}$ and $B_{c}$ discretizing the zero mean value constraint.

\subsection{Numerical investigations}

\subsubsection{Test case setup}

The efficiency of the hybrid model is illustrated in this section. For this an anisotropy ratio with large variations within the computational domain is constructed thanks to the following definition

$$
\varepsilon(z)=\frac{1}{2}\left(\varepsilon_{\max }(1+\tanh (r z))+\varepsilon_{\min }(1-\tanh (r z))\right),
$$

where $\varepsilon_{\min }>0$ and $\varepsilon_{\max }>0$ define the range of values covered by $\varepsilon, r \in \mathbb{R}$ defining the rate of change. In the sequel this parameter value is set to $r=30$. The different 


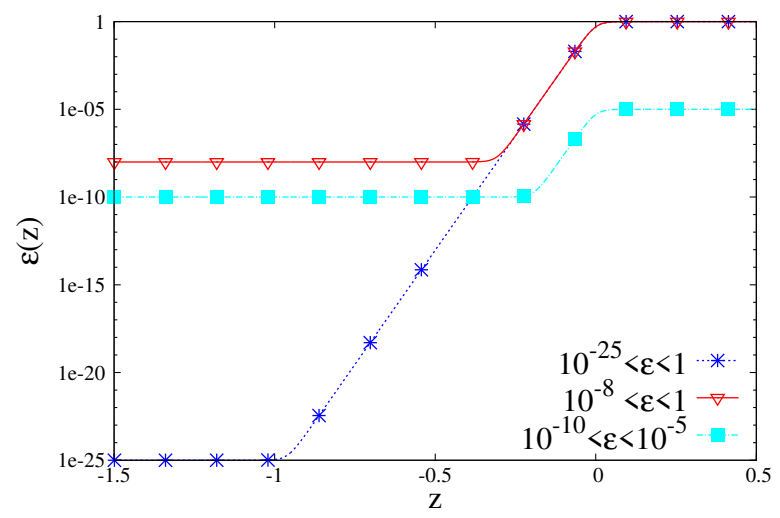

Figure 2: Anisotropy ratio $\varepsilon$ as a function of $z$, for different values of $\varepsilon_{\min }$ and $\varepsilon_{\max }$ with $r=30$.

heterogeneous anisotropy ratios used for the numerical investigations are represented on Figure 2 as a function of $z$.

The diffusion coefficients are defined as

$$
A_{x}(x, z)=c_{1}+x z^{2}, \quad A_{z}(x, z)=c_{2}+x z,
$$

with $c_{1} \in \mathbb{R}, c_{2} \in \mathbb{R}$ two constants chosen to meet the requirements $A_{x}(x, z) \geq m_{x}>0$ and $A_{z}(x, z) \geq m_{z}>0$ for $(x, z) \in \Omega$.

With these definitions an analytic setup is manufactured thanks to the exact solution

$$
u_{e}(x, z)=\sin \left(\frac{2 \pi}{L_{x}} x\right)\left(1+\varepsilon(z) \sin \left(\frac{2 \pi}{L_{z}} z\right)\right),
$$

by inserting this expression into (1.1)-(1.2) for the computations of the second member of the problem $f$ as well as $g_{+}$and $g_{-}$. A second setup is investigated with the following definitions for the diffusion coefficients

$$
A_{x}(x, z)=1+\cos \left(c_{1}+x z\right), \quad A_{z}(x, z)=1+\sin ^{2}\left(c_{2}+x z\right),
$$

and the exact solution

$$
u_{e}(x, z)=\sin \left(\frac{2 \pi}{L_{x}} x\right)\left(1+\sin \left(\frac{2 \pi}{L_{z}} \varepsilon(z) z\right)\right) .
$$

The simulations are performed with $c_{1}=c_{2}=L_{z}$, on the domain $\Omega_{a}=\Omega_{x} \times \Omega_{z}=$ $[0,1] \times[-1,1]$ for homogeneous $\varepsilon$ and with $\Omega_{b}=\Omega_{x} \times \Omega_{z}=[0,1] \times[-1.5,0.5]$ for $\varepsilon$ defined by (4.47). The results obtained for both setups (4.48)-(4.49) and (4.50)-(4.51) being very similar, only the computations performed thanks to the first definitions are reported in the sequel.

The linear systems stemming from the discretization of the singular perturbation problem (2.5), the AP-model (2.10) and the hybrid (AP/L)-model (3.17) are solved using the same solver. For the computations carried out in this paper, the sparse direct solver MUMPS 22] is used. 


\subsubsection{Choice of the interface}

The choice of the interface location $z_{\iota}$ is of great importance because it influences the performance of the scheme, in terms of precision and computational time.

This question is often pointed out in hybrid models based on domain decomposition strategies. As explained in the introduction and the beginning of section 3 , the motivation of our $(\mathrm{AP} / \mathrm{L})$-coupling strategy is that it is always possible to situate the interface, which is not always the case in a $(\mathrm{P} / \mathrm{L})$-coupling method. The position of the interface has to be chosen on one hand in order to preserve the precision of the numerical scheme and on the other hand to achieve a gain in the computational time (i.e. largest possible $L$-sub-domain). This means that the approximation error between the AP-solution and the hybrid (AP/L)-solution has to be of the order of the precision of the numerical scheme.

The analytic investigations carried out in section 3 (see Theorem 3.3) demonstrate that the error produced by the use of the limit problem in one sub-domain is bounded by the value of the asymptotic parameter at the interface, i.e. $\sqrt{\varepsilon\left(z_{\iota}\right)}$. If we denote by $u$ the exact solution of the problem, by $v$ the exact solution of the hybrid model and finally by $v_{h}$ its numerical approximation, the following inequality $\left\|u-v_{h}\right\|_{H^{1}} \leq$ $\|u-v\|_{H^{1}}+\left\|v-v_{h}\right\|_{H^{1}}$ holds, leading to

$$
\left\|u-v_{h}\right\|_{H^{1}} \leq c \sqrt{\varepsilon\left(z_{\iota}\right)}+C h^{m}
$$

$C>0$ denoting a constant, $h$ the typical mesh size and $m$ the approximation order of the numerical methods defined as the convergence rate of the error in the $H^{1}$-norm. In order to prevent any deterioration of the numerical method precision, the interface should hence be located in a region of the computational domain where the following condition is met $\sqrt{\varepsilon\left(z_{\iota}\right)} \sim h^{m}$.

To verify numerically the statements of Theorem 3.3 and more particularly the error estimate provided by the equation (4.52), a series of computations have been run. The evolution of the error measured between the exact solution (manufactured thanks to the set-up (4.48) and (4.49)) and the approximation carried out thanks to the hybrid method is displayed on Figure 3 (left plots). This Figure displays also the value of the optimal asymptotic parameter at the interface denoted $\varepsilon\left(z_{\iota}^{\star}\right)$ as a function of the mesh size. The optimal value of $\varepsilon\left(z_{\iota}\right)$ is the largest value preserving the accuracy of the numerical method. As reported on Figure 3, the approximation error is decreasing with $\varepsilon\left(z_{\iota}\right)$ until a plateau is reached. Above this $\varepsilon\left(z_{\iota}^{\star}\right)$-value, the error is dominated by the modeling error, due to the use of the limit model for large values of the asymptotic parameter. Below this value, the error does not depend on $\varepsilon\left(z_{\iota}\right)$ anymore and reduces to the numerical approximation error proportional to $h^{m}$. This optimal value ensures that the limit model is used on the largest sub-domain as possible without introducing any discrepancy in the numerical method precision. The second plot displays $\varepsilon\left(z_{\iota}^{\star}\right)$ as a function of the mesh size. A linear decrease of the $\varepsilon\left(z_{\iota}^{\star}\right)$ values with the mesh size is observed, analogously to the decrease of the error $H^{1}$-norm with the $\varepsilon\left(z_{\iota}\right)$-values, while this decrease is expected, as proved in Theorem 3.3 and stated in the equation (4.52) to be proportional to $\sqrt{\varepsilon\left(z_{\iota}\right)}$. This test case has been reproduced with different set-ups obtaining the same decrease rate of the approximation error. However, the $\sqrt{\varepsilon\left(z_{\iota}\right)}$ estimate of Theorem 3.3 is the only estimate we can guarantee such that the condition 

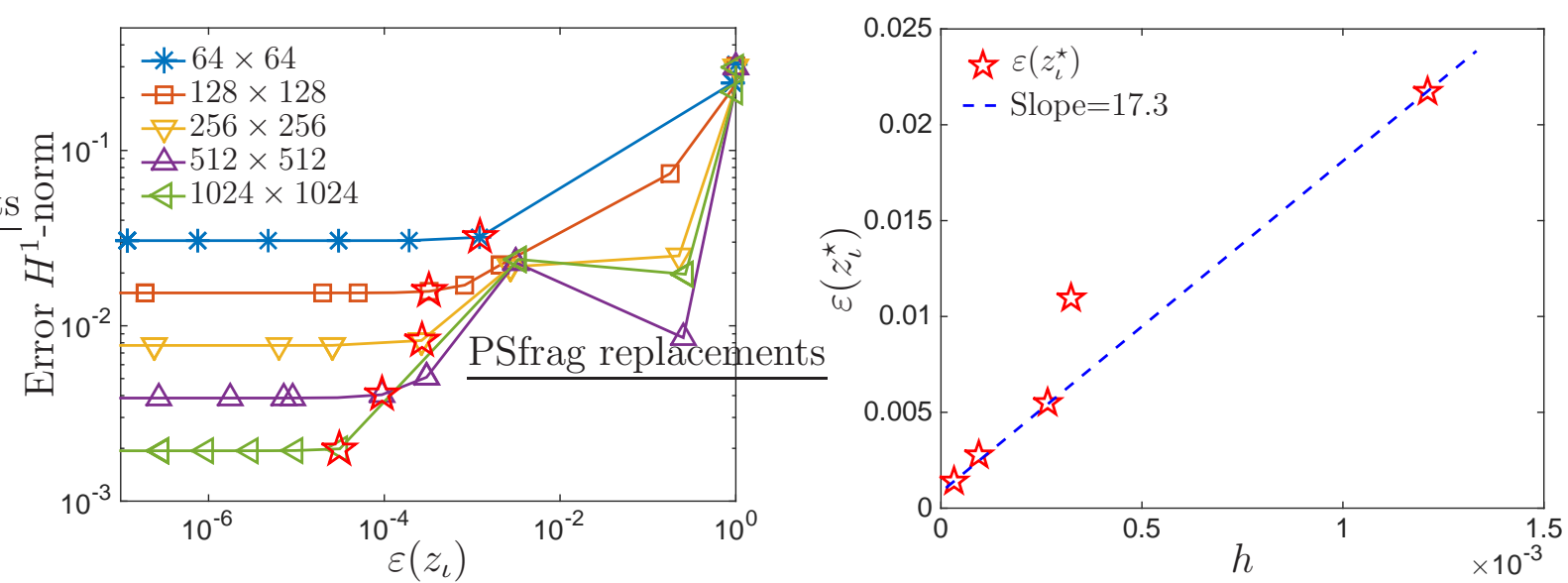

Figure 3: Error $H^{1}$-norm between the exact solution and the approximation computed by the $(\mathrm{AP} / \mathrm{L})$-scheme on different meshes (left), the optimal value of $\varepsilon\left(z_{\iota}\right)$ denoted $\varepsilon\left(z_{\iota}^{\star}\right)$ is identified by a pentagram marker; Optimal value of $\varepsilon\left(z_{\iota}\right)$ as a function of the mesh size $h$ (right).

$\sqrt{\varepsilon\left(z_{\iota}\right)} \sim h^{m}$ should be used to locate the interface.

This hybrid method has been developed to accelerate the simulation of time dependent systems described by a set of equations, in which the anisotropic elliptic equation investigated here provides one of the unknowns. This system is assumed to incorporate two different time scales. The first one is related to the evolution of the quantities advanced by the set of equations. The second one, much slower, characterizes the variations of the anisotropy ratio. This is the typical framework for the simulation of ionospheric plasma disturbances already mentioned in the introduction. Indeed the dynamics of the plasma perturbations is fast compared to that of the neutral particles properties that are responsible for the anisotropy variations. In this framework, the interface can be precisely located at the initial time, thanks to the use of the AP-scheme providing a reference solution. Note that the implementation of the AP-scheme is readily obtained from that of the hybrid model. Then the computation of a series of time steps can be accelerated using the hybrid model for the anisotropic equation resolution. When the anisotropy ratio value undergoes significant variations, the procedure can be repeated to determine a new location for the interface. The hybrid method is thus all the more efficient than the time scales separation is large.

Remark 4.1 The hybrid model requires two sub-domains verifying: $\Omega_{z}^{1} \neq \emptyset$ and $\Omega_{z}^{2} \neq \emptyset$. This means that for $\varepsilon \sim \mathcal{O}(1)$ uniformly in the domain, the method introduced here cannot provide accurate computations since the limit problem will be used in one part of the domain where the asymptotic parameter is large. In such a situation the P-model or the AP-formulation should be preferred.

\subsubsection{Accuracy of the numerical method}

The precision of the hybrid numerical method is first examined. Primarily, we represent the solution computed by the (AP/L)-scheme when $\varepsilon$ is given by (4.47) with $\varepsilon_{\text {min }}=10^{-8}$, $\varepsilon_{\max }=1, N_{x}=N_{z}=64$ on Figure 4(a). We also give the difference between this 
computed solution and the exact one on Figure 4(b)

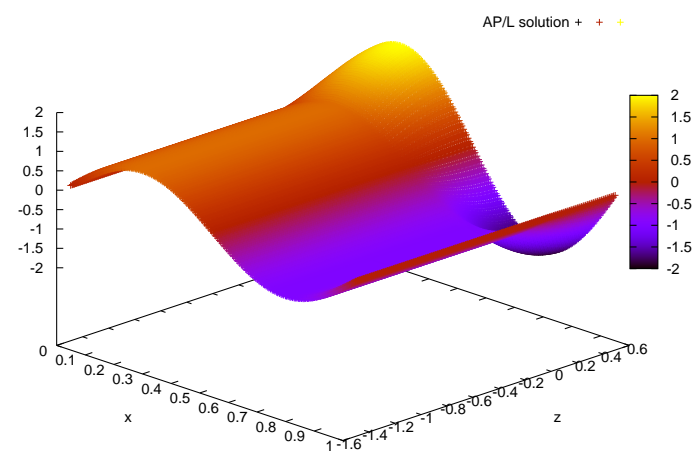

(a)

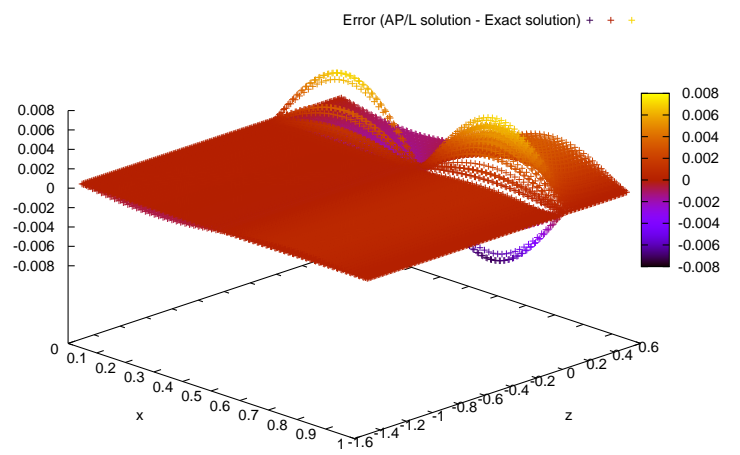

(b)

Figure 4: $\varepsilon$ given by (4.47) case, with $\varepsilon_{\min }=10^{-8}, \varepsilon_{\max }=1$ : (a) solution of the $(\mathrm{AP} / \mathrm{L})$-scheme, (b) difference with the exact solution.

The relative $\mathrm{H}^{1}$-error between the exact solution (4.49) and its numerical approximation via the $(\mathrm{AP} / \mathrm{L})$-model is plotted on Figure 5(a) as a function of the mesh size $h:=(\Delta x \Delta z)^{\frac{1}{2}}$. Note that the computations are performed with the same number of cells in each direction, which amounts, accordingly to the setup precised in section 4.2.1, to $\Delta z=2 \Delta x$. These errors are computed first, for constant $\varepsilon$, with $\varepsilon$ ranging from $10^{-25}$ to $10^{-8}$ (Figure $5(\mathrm{a})$. Second, the same computations are performed and plotted on Figure 5(b) for heterogeneous anisotropy ratios $\varepsilon$ defined by (4.47) with $\varepsilon_{\max }$ set to 1 and $\varepsilon_{\min }$ ranging from $10^{-25}$ to $10^{-8}$ as displayed on Figure 2 (curves with triangle and star markers). The reference solution is manufactured thanks to the setup (4.48)-(4.49).

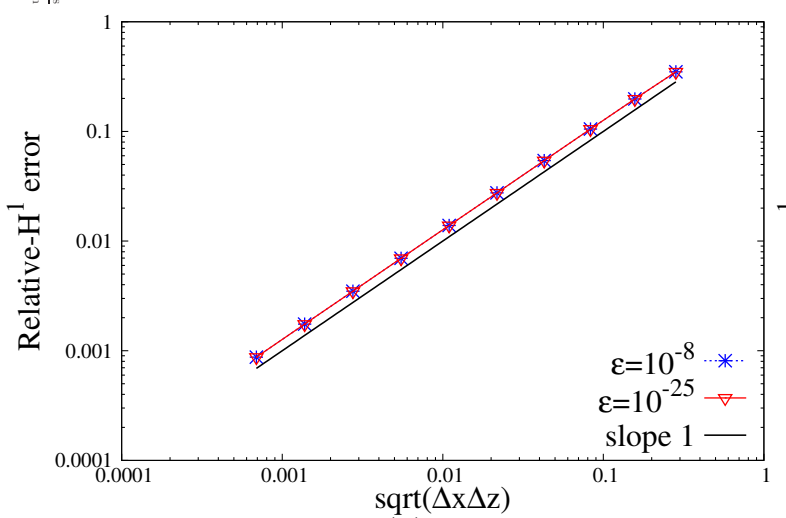

(a)

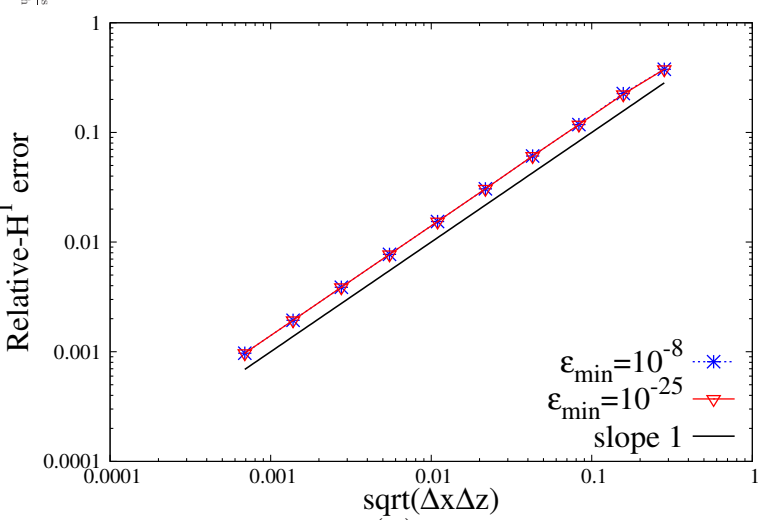

(b)

Figure 5: $\mathrm{H}^{1}$ relative error between the exact solution and its numerical approximation as a function of the mesh size $h:=(\Delta x \Delta z)^{\frac{1}{2}}$ : (a) accuracy for constant anisotropy ratios $\varepsilon=10^{-8}$ and $\varepsilon=10^{-25}$, (b) accuracy for heterogeneous anisotropy ratios defined by (4.47) with $\varepsilon_{\max }=1$, and $\varepsilon_{\min }=10^{-8}$ or $\varepsilon_{\min }=10^{-25}$. 
The hybrid method is observed to be first order accurate for both sets of computations as soon as the interface is located in a region where the value of $\varepsilon$ is small enough compared to the precision of the spatial discretization.

\subsubsection{Asymptotic preserving property of the hybrid model}

The Asymptotic-Preserving property of the hybrid model is now investigated. The error between the solution and its numerical approximations computed thanks to the hybrid (AP/L)-model (3.17), the standard AP-scheme (2.10) and the discretized singular perturbation problem (2.5), as well as the condition number of the corresponding linear systems, are plotted on Figures 6 and 7 . These computations are carried out on meshes with $64 \times 64$ and $1024 \times 1024$ cells with the heterogeneous anisotropy ratio defined by (4.47). The condition numbers reported on these figures are estimated by the MUMPS solver. Concerning the condition number, remark that the relative error of the linear system solution, carried out by the solver, is upper bounded by two parameters denoted by $\mathrm{CN}_{1}$ and $\mathrm{CN}_{2}$ (see subsection 2.3 of [22]) and plotted on Figures 6(b) and 7(b).

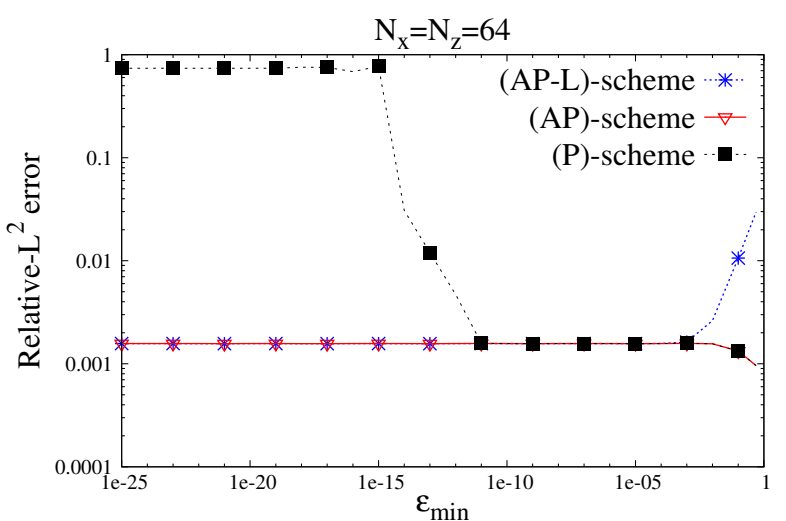

(a)

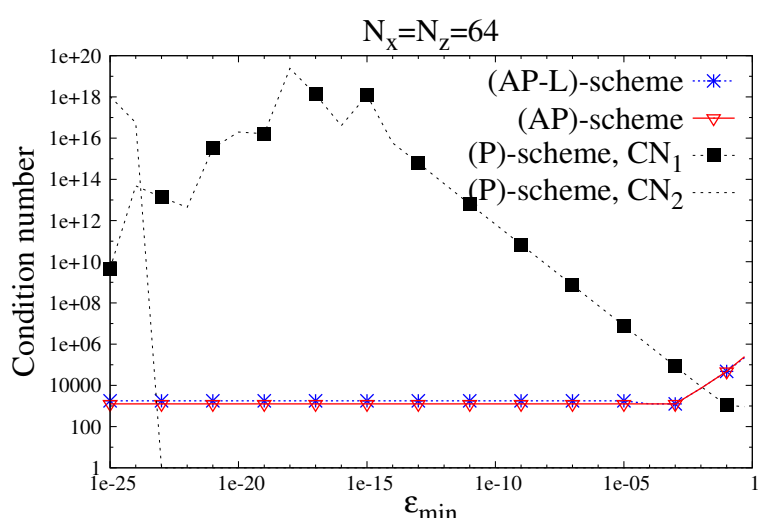

(b)

Figure 6: Comparison of the hybrid (AP/L)-model, the AP-scheme and the singular perturbation P-model accuracy for heterogeneous anisotropy ratios as defined by (4.47) on a $64 \times 64$ mesh: (a) relative $\mathrm{L}^{2}$-error between the exact solution and its numerical approximations and (b) condition number of the linear systems (estimated by MUMPS) as a functions of $\varepsilon_{\min }\left(\right.$ with $\varepsilon_{\max }=1$ ).

The condition number of the singular perturbation problem increases almost linearly with vanishing $\varepsilon_{\min }$. When the condition number is comparable to the computer arithmetic precision, the accuracy of the solution is significantly altered as depicted on Figures 6(a) and $7(\mathrm{a})$. On the coarsest mesh the accuracy of the computations is altered for $\varepsilon_{\min }$-values smaller than $10^{-11}$ (see Figure 6(b). For these values the condition number of the linear system is reported by the solver to be as large as $10^{13}$. For the computations carried out on the refined mesh, this threshold is reached for larger values of the $\varepsilon_{\text {min }}$, precisely $\varepsilon_{\min }=10^{-7}$, corresponding to an estimate of condition number value above $10^{12}$. On this refined mesh, the solution cannot be accurately approximated via the singular perturbation problem for variations of the anisotropy ratio larger than 


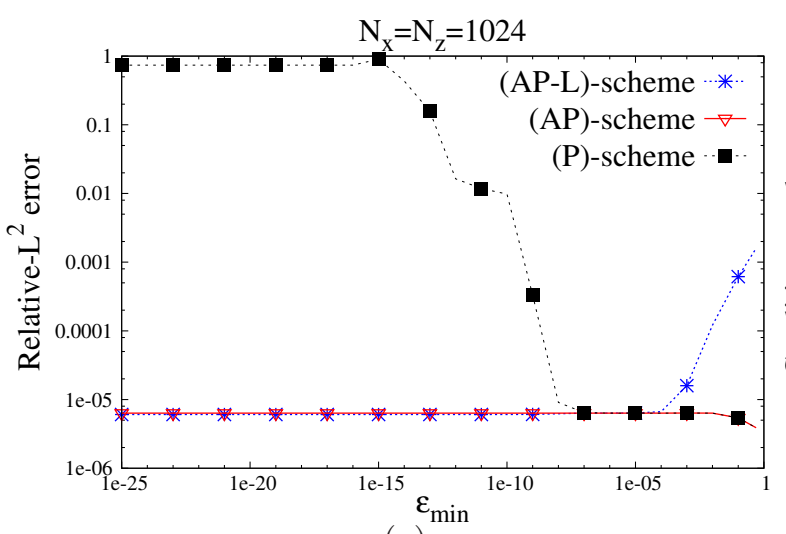

(a)

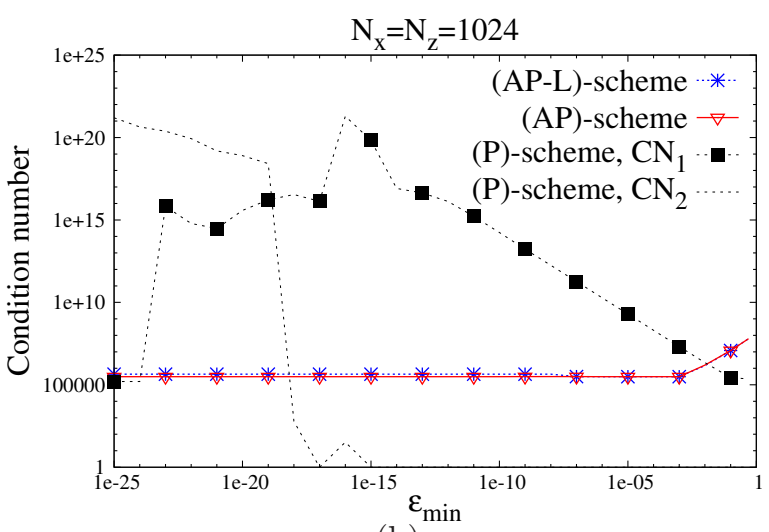

(b)

Figure 7: Comparison of the hybrid (AP/L)-model, the AP-scheme and the singular perturbation P-model accuracy for heterogeneous anisotropy ratios as defined by (4.47) on a $1024 \times 1024$ mesh: (a) relative $\mathrm{L}^{2}$-error between the exact solution and its numerical approximations and (b) condition number of the linear systems (estimated by MUMPS) as a functions of $\varepsilon_{\min }\left(\right.$ with $\varepsilon_{\max }=1$ ).

$10^{7}$, as depicted on Figure 7(a). The range of anisotropy variations tractable by the $\mathrm{P}$-model is getting narrower with the mesh refinement.

The AP property on the standard AP-scheme translates into a condition number as well as a precision almost independent of the anisotropy strength.

As for the hybrid model, the condition number is observed to be very similar to that of the standard AP-scheme, with almost no variations with respect to the anisotropy strength. Concerning the precision, two regimes can be identified. For small enough

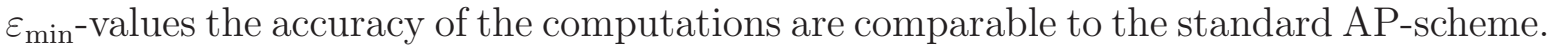
For computations with $\varepsilon_{\text {min }}$-values that are not so small (see the growth of the error on Figures $6(\mathrm{a})$ and $7(\mathrm{a})$ for the largest values of $\varepsilon_{\min }$ ), the limit regime is used in a subdomain where the asymptotic parameter is too large to guarantee a good approximation of the solution. The approximation error explained by the use of the limit problem in one sub-domain is thus larger than the numerical error of the discretization, producing computations with a poor accuracy. Since the precision of the space discretization increases with vanishing mesh sizes, the accuracy of the computations carried out by the hybrid model is improved by a mesh refinement if the coupling interface is immersed in an area where the value of the asymptotic parameter is small enough. For the coarsest mesh, this requirement is met for $\varepsilon_{\min }$ values smaller than $10^{-3}$ while this threshold equals $10^{-4}$ for the refined mesh.

\subsubsection{Efficiency of the hybrid numerical method}

Finally, the numerical efficiency of the hybrid method (in terms of simulation time and memory usage) is compared to that of the fully AP-scheme and the discretized singular perturbation problem. Two different sets of computations have been performed. The first one is related to an anisotropy ratio defined by (4.47) with $\varepsilon_{\max }=1$ and $\varepsilon_{\min }=10^{-8}$ and with a domain decomposition verifying $\left|\Omega_{z}^{1}\right|=2 / 5\left|\Omega_{z}\right|(|\cdot|$ denoting the length) and 
$\left|\Omega_{z}^{2}\right|=3 / 5\left|\Omega_{z}\right|$. For the second setup the sub-domain $\Omega_{z}^{2}$ is enlarged with a decomposition yielding to $\left|\Omega_{z}^{1}\right|=3 / 10\left|\Omega_{z}\right|$ and $\left|\Omega_{z}^{2}\right|=7 / 10\left|\Omega_{z}\right|$. The anisotropy ratio obeys the same definition (4.47) but with $\varepsilon_{\max }=10^{-5}$ and $\varepsilon_{\min }=10^{-10}$. The main characteristics of the computations are gathered in Table1for the first setup and in Table 2 for the second one.

\begin{tabular}{|c|c|r|r|r|r|r|}
\hline Scheme & $N_{x}=N_{z}$ & Time (AMF) & \#entries in factor (AMF) & \#rows & \#non zeros & L $^{2}$-error \\
\hline \hline $\mathrm{AP} / \mathrm{L}$ & 250 & $83 \%(72 \%)$ & $3705458(2859836)$ & 26000 & 533324 & $1.06 \times 10^{-4}$ \\
\hline $\mathrm{AP}$ & 250 & $247 \%(187 \%)$ & $10742572(9301072)$ & 63500 & 1318724 & $1.06 \times 10^{-4}$ \\
\hline $\mathrm{P}$ & 250 & $100 \%(100 \%)$ & $5614870(5726236)$ & 63000 & 563992 & $1.06 \times 10^{-4}$ \\
\hline \hline $\mathrm{AP} / \mathrm{L}$ & 500 & $83 \%(72 \%)$ & $18461132(13736886)$ & 102000 & 2116674 & $2.64 \times 10^{-5}$ \\
\hline $\mathrm{AP}$ & 500 & $311 \%(219 \%)$ & $61429052(40562916)$ & 252000 & 5262474 & $2.65 \times 10^{-5}$ \\
\hline $\mathrm{P}$ & 500 & $100 \%(100 \%)$ & $26697996(26940422)$ & 251000 & 2252992 & $2.70 \times 10^{-5}$ \\
\hline \hline $\mathrm{AP} / \mathrm{L}$ & 1000 & $72 \%(61 \%)$ & $85235506(61449488)$ & 404000 & 8433374 & $6.52 \times 10^{-6}$ \\
\hline $\mathrm{AP}$ & 1000 & $357 \%(213 \%)$ & $319347622(183083450)$ & 1004000 & 21024974 & $6.64 \times 10^{-6}$ \\
\hline $\mathrm{P}$ & 1000 & $100 \%(100 \%)$ & $130031284(121110920)$ & 1002000 & 9005992 & $2.23 \times 10^{-5}$ \\
\hline \hline $\mathrm{AP} / \mathrm{L}$ & 2000 & $74 \%(43 \%)$ & $527455320(289307676)$ & 1608000 & 33666774 & $1.54 \times 10^{-6}$ \\
\hline $\mathrm{AP}$ & 2000 & $351 \%(146 \%)$ & $1678279706(802178884)$ & 4008000 & 84049974 & $1.66 \times 10^{-6}$ \\
\hline $\mathrm{P}$ & 2000 & $100 \%(100 \%)$ & $600370134(557859738)$ & 4004000 & 36011992 & $8.88 \times 10^{-5}$ \\
\hline
\end{tabular}

Table 1: Efficiency of the Hybrid and AP methods compared to the discretized singular perturbation model: computational time relative to that of the P-model with the MUMPS solver and the METIS (AMF) ordering, number of non zero elements after factorization with the METIS (AMF) ordering, number of rows and of non zero elements in the matrices, and precision of the computations (relative error $\mathrm{L}^{2}$-norm) carried out with $\left|\Omega_{z}^{1}\right|=2 / 5\left|\Omega_{z}\right|$ and $\left|\Omega_{z}^{2}\right|=3 / 5\left|\Omega_{z}\right|$ on different mesh resolutions.

Columns 5 and 6 display the number of rows and the number of non zero elements in the matrices stemming from the discretization of the three methods. The computational time required to solve these linear systems using the same solver (MUMPS) with two different matrix ordering algorithms (METIS [18] and AMF [24]) is collected in column 3. The entries of this column are relative to the computational time of the P-model. The number of non zero elements in the factorized matrix is precised in column 4 for the two matrix orderings mentioned above. The last column gives the relative $\mathrm{L}^{2}$-error between the exact solution and the numerical approximations. The numerical method is specified in the first column and the mesh size in the second one.

The AP-scheme requires a larger memory amount than the P-model to store the linear system matrix. This growth is marginally explained by the increase in the number of unknowns due to the computation of the solution mean part, on the one hand, and to the introduction of the Lagrangian, on the other hand. Fortunately, both unknowns only depend on the $x$ coordinate and explain thus a moderate increase in the number of the matrix rows : from $N_{x} \times\left(N_{z}+2\right)$ for the P-model to $\left(N_{x} \times\left(N_{z}+4\right)\right)$ for the AP-scheme. However, the AP-scheme matrix exhibits an amount of non zero elements 


\begin{tabular}{|c|c|r|r|r|r|r|}
\hline Scheme & $N_{x}=N_{z}$ & Time (AMF) & \#entries in factor (AMF) & \#rows & \#non zeros & L $^{2}$-error \\
\hline \hline $\mathrm{AP} / \mathrm{L}$ & 250 & $57 \%(56 \%)$ & $2431654(1999636)$ & 19750 & 402424 & $5.61 \times 10^{-5}$ \\
\hline $\mathrm{AP}$ & 250 & $232 \%(213 \%)$ & $10737412(9301072)$ & 63500 & 1318724 & $5.61 \times 10^{-5}$ \\
\hline $\mathrm{P}$ & 250 & $100 \%(100 \%)$ & $5614870(5728812)$ & 63000 & 563992 & $1.51 \times 10^{-4}$ \\
\hline \hline $\mathrm{AP} / \mathrm{L}$ & 500 & $58 \%(53 \%)$ & $13975832(9861960)$ & 77000 & 1592374 & $1.41 \times 10^{-5}$ \\
\hline $\mathrm{AP}$ & 500 & $301 \%(203 \%)$ & $61469878(40655248)$ & 252000 & 5262474 & $1.41 \times 10^{-5}$ \\
\hline $\mathrm{P}$ & 500 & $100 \%(100 \%)$ & $26699956(26940422)$ & 251000 & 2252992 & $3.35 \times 10^{-3}$ \\
\hline \hline $\mathrm{AP} / \mathrm{L}$ & 1000 & $43 \%(39 \%)$ & $60103598(45148064)$ & 304000 & 6334774 & $3.58 \times 10^{-6}$ \\
\hline $\mathrm{AP}$ & 1000 & $329 \%(190 \%)$ & $320132040(183805436)$ & 1004000 & 21024974 & $3.53 \times 10^{-6}$ \\
\hline $\mathrm{P}$ & 1000 & $100 \%(100 \%)$ & $130031284(121110920)$ & 1002000 & 9005992 & $1.21 \times 10^{-2}$ \\
\hline \hline $\mathrm{AP} / \mathrm{L}$ & 2000 & $41 \%(26 \%)$ & $351816570(206531976)$ & 1208000 & 25269574 & $1.17 \times 10^{-6}$ \\
\hline $\mathrm{AP}$ & 2000 & $299 \%(137 \%)$ & $1682372230(804867106)$ & 4008000 & 84049974 & $8.84 \times 10^{-7}$ \\
\hline $\mathrm{P}$ & 2000 & $100 \%(100 \%)$ & $600370134(557859738)$ & 4004000 & 36011992 & $1.50 \times 10^{-2}$ \\
\hline
\end{tabular}

Table 2: Efficiency of the Hybrid and AP methods compared to the discretized singular perturbation model: computational time relative to that of the P-model with the MUMPS solver and the METIS (AMF) ordering, number of non zero elements after factorization with the METIS (AMF) ordering, number of rows and of non zero elements in the matrices, and precision of the computations (relative error $\mathrm{L}^{2}$-norm) carried out with $\left|\Omega_{z}^{1}\right|=3 / 10\left|\Omega_{z}\right|$ and $\left|\Omega_{z}^{2}\right|=7 / 10\left|\Omega_{z}\right|$ on different mesh resolutions.

$\left(\left(7 N_{z}+13\right)\left(3 N_{x}-2\right)\right.$, see also [2, Figure 1]) significantly increased, by a factor of about $7 / 3 \approx 2.33$, compared to that of the P-model $\left(\left(3 N_{z}+4\right)\left(3 N_{x}-2\right)\right)$. This is equally explained by the $B_{l}$ and $B_{c}$ sub-matrices, introduced in (4.45) for the discretization of the fluctuation zero mean value constraint, and by the coupling between the mean and the fluctuating parts of the solution by means of the $C_{a}$ and $C_{f}$ sub-matrices. These sub-matrices have a large amount of non zero elements compared to the $A_{x a}$ sub-matrix and explain thus most of the increase in the AP-scheme memory usage. A similar conclusion can be drawn from the comparisons of the factorized matrices. The fill-in of the factorized AP-matrix ranges from $140 \%$ to $280 \%$ that of the P-model (column 4 of Tables [1] and 2).

The improvement in the hybrid method efficiency is obtained thanks to the reduction of the fluctuation computational domain size. This offers a significant decrease in the row number of the hybrid (AP/L)-model matrix (4.44) as well as a reduction in the size of the sub-matrices with the poorest sparsity. Compared to the AP-scheme, the number of non zero elements stored in the (AP/L)-matrix is roughly divided by 2.4 for the first setup (Table 1) and by 3.3 for the second one (Table 21). Compared to the P-model, a gain in the matrix size of the $(\mathrm{AP} / \mathrm{L})$-scheme can be anticipated as soon as the interface position verifies $\iota>4 / 7 N_{z} \approx 0.57 N_{z}$. The fill-in is reduced by $5 \%$ to $9 \%$ for the first setup $\left(\iota=3 / 5 N_{z}\right)$ and $20 \%$ to $30 \%$ for the second one $\left(\iota=7 / 10 N_{z}\right)$. Importantly also for the linear system solver used for these investigations, this advantage is amplified for the 
sparsity of the factorized matrix. For the most favorable setup, the fill-in of the (AP/L) factorized matrix is only $43 \%$ to $59 \%$ that of the P-model matrix for the METIS ordering and $26 \%$ to $56 \%$ for the AMF algorithm. This gain results in a significant reduction of the computation time with a speed-up, relatively to the P-model, ranging from 1.2 to 3.8 over the data collected in these investigations.

Finally the last column of Tables 1 and 2 illustrates the precision of the different methods. For the first setup, both AP-methods produce accurate computations with a small advantage for the hybrid method (at most, less than 10\%, for the largest system size). For the second setup, the hybrid (AP/L)-method fails to reach the accuracy of the standard AP-method for the most refined meshes. The mesh refinement increases the precision of the space discretization and necessitates, in order to improve the overall method accuracy, an interface immersed in a region with small enough $\varepsilon$-values. This requirement is met for the first setup, but not for the two most refined meshes with the second one. Note that the accuracy of the hybrid (AP/L)-method may be recovered by moving the interface location into a region with smaller $\varepsilon$-values.

These numerical investigations demonstrate a significant advantage of the hybrid method as soon as the limit model can be used on a sub-domain large enough. For the numerical investigations performed in this section, the overhead of the AP-scheme is completely erased. In favorable situations, the hybrid method is more efficient in terms of storage requirements as well as in the computational time than the standard Pmodel. Of course, these results should be worsen or improved accordingly to the domain decomposition authorized by the anisotropy ratio values and the precision required.

\section{Conclusion}

In this paper, we studied a 2D highly anisotropic elliptic problem, whose initial formulation (called P-model) leads to an ill-conditioned system when the anisotropy parameter $\varepsilon$ tends to zero. Degond et al. developed recently an asymptotic-preserving AP-model to overcome this problem (see [7] for example). It is uniformly accurate for any value of $\varepsilon \in[0,1]$, but more costly than the P-model.

In the context of ionospheric plasmas, $\varepsilon$ appears to be very small in a large region of the domain, such that, in this region, the use of the 1D limit L-model can be interesting from a computational point of view. The coupling of the L-model to the P-model is not always possible, because their validity domains may not overlap. That is the reason why we proposed to solve the L-model in the region where $\varepsilon$ is close to zero, while an AP-model is used in the rest of the domain. The two models are coupled thanks to Dirichlet-Neumann transfer conditions.

The coupling strategy has been detailed and mathematically analyzed. Then, we have presented the numerical discretization and applied our new method in some test cases. The obtained (AP/L)-model is shown to be accurate and well-conditioned even if $\varepsilon$ presents steep gradients with values closed to zero in one part of the domain and of order one in the other. Moreover, the computational time is significantly reduced compared to the full AP-model, due to the use of the 1D L-model in a large part of the domain. 
Acknowledgement. F. Deluzet and C. Negulescu would like to thank Pierre Degond for having initiated this series of works on highly anisotropic elliptic equations. This work has been supported by the Agence Nationale de la Recherche (ANR) under contract IODISSEE (IOnospheric DIsturbanceS and SatEllite-to-Earth communications, ANR-09-COSI-007-02) and the FR-FCM (Fédération de Recherche pour la Fusion par Confinement Magnétique).

\section{References}

[1] C. Besse, J. Claudel, P. Degond, F. Deluzet, G. Gallice, C. Tessieras, A model hierarchy for ionospheric plasma modeling, Mathematical Models \& Methods in Applied Sciences 14 (2004), no. 3.

[2] C. Besse, F. Deluzet, C. Negulescu, C. Yang, Efficient numerical methods for strongly anisotropic elliptic equations, Journal of Scientific Computing 55 (2013), no. $1,231-254$.

[3] H. Brezis, Functional Analysis, Sobolev Spaces and Partial Differential Equations, Universitext, Springer (2010).

[4] F.F. Chen, Introduction to Plasma Physics and Controlled Fusion: Plasma Physics, Springer (1984).

[5] P. Degond, F. Deluzet, A. Lozinski, J. Narski, C. Negulescu, Duality based Asymptotic-Preserving Method for highly anisotropic diffusion equations, Communications in Mathematical Sciences 10 (2012), no. 1, 1-31.

[6] P. Degond, F. Deluzet, D. Maldarella, J. Narski, C. Negulescu, M. Parisot, Hybrid model for the coupling of an Asymptotic Preserving scheme with the asymptotic limit model: the one dimensional case, ESAIM Proc. 32 (2011), 23-30.

[7] P. Degond, F. Deluzet, C. Negulescu, An Asymptotic Preserving scheme for strongly anisotropic elliptic problem, SIAM Multiscale Modeling and Simulation 8 (2010), no. 2, 645-666.

[8] P. Degond, S. Jin, A Smooth Transition Model Between Kinetic and Diffusion Equations, SIAM Journal on Numerical Analysis 42 (2004), no. 6, 2671-2687.

[9] P. Degond, S. Jin, L. Mieussens, A smooth transition model between kinetic and hydrodynamic equations, J. Comput. Phys. 209 (2005), 665-694.

[10] P. Degond, A. Lozinski, J. Narski, C. Negulescu, An Asymptotic-Preserving method for highly anisotropic elliptic equations based on a micro-macro decomposition, Journal of Computational Physics 231 (2012), no. 7, 2724-2740.

[11] P. Degond, C. Schmeiser, Kinetic boundary layers and fluid-kinetic coupling in semiconductors, Transport Theory Statist. Phys. 28 (1999), 31-55. 
[12] L. C. Evans, Partial Differential Equations, Volume 19 of Graduate Studies in Mathematics, ISSN 1065-7339, American Mathematical Society (2010).

[13] R.J. Goldston, P.H. Rutherford, Introduction to Plasma Physics, CRC Press (1995).

[14] W.J. Heikkila, Earth's Magnetosphere: Formed by the Low-latitude Boundary Layer, Elsevier (2011).

[15] J.D. Huba, G. Joyce, J. Krall, Three-Dimensional Modeling of Equatorial Spread F, in: Abdu, M.A., Pancheva, D. (Eds.), Aeronomy of the Earths Atmosphere and Ionosphere, IAGA Special Sopron Book Series. Springer Netherlands (2011), $211-218$.

[16] S.C. Jardin, Review of implicit methods for the magnetohydrodynamic description of magnetically confined plasmas, Journal of Computational Physics 231 (2012), no. $3,822-838$.

[17] S. Jin, Efficient asymptotic-preserving (AP) schemes for some multiscale kinetic equations, SIAM Journal on Scientific Computing 21 (1999), no. 2, 441-454.

[18] G. Karypis, V. Kumar, METIS A Software Package for Partitioning Unstructured Graphs, Partitioning Meshes, and Computing Fill-Reducing Orderings of Sparse Matrices Version 4.0., University of Minnesota (1998).

[19] A. Klar, Asymptotic Induced Domain Decomposition Methods for Kinetic and Drift Diffusion Semiconductor Equations, SIAM J. Sci. Comp. 19 (1998), no. 6, 20322050 .

[20] A. Klar, H. Neunzert, J. Struckmeier, Transition from Kinetic Theory to Macroscopic Fluid Equations: A Problem for Domain Decomposition and a Source for New Algorithms, TTSP 29 (2000), no. 1-2, 93-106.

[21] J. Krall, J.D. Huba, G. Joyce, S.T. Zalesak, Three-dimensional simulation of equatorial spread-F with meridional wind effects. Annales Geophysicae 27 (2009), 18211830 .

[22] MUltifrontal Massively Parallel Solver (MUMPS 4.10.0), Users' guide (2011).

[23] A. Quarteroni, A. Valli, Domain decomposition methods for partial differential equations, Oxford University Press, New York (1999).

[24] E. Rothberg, Ordering sparse matrices using approximate minimum local fill, Second SIAM Conference on Sparse Matrices (1996).

[25] R.W. Schunk, A. Nagy, Ionospheres: Physics, Plasma Physics, and Chemistry, Cambridge University Press (2004).

[26] T.H. Stix, Waves in Plasmas, American Institute of Physics, New York (1992).

[27] C. Yang, Analyse et mise en oeuvre des schémas numériques pour la physique des plasmas ionosphériques et de tokamaks, PhD thesis, Université Lille 1 Sciences et Technologies (2011). 